\title{
Unique solvability of compressible micropolar viscous fluids
}

Mingtao Chen

Correspondence: mtchen@sdu.edu. $\mathrm{cn}$

School of Mathematics and

Statistics, Shandong University at Weihai, Weihai 264209, PR China

\section{Abstract}

In this article, we consider the compressible micropolar viscous flow in a bounded or unbounded domain $\Omega \subseteq \mathbb{R}^{3}$. We prove the existence of unique local strong solutions for large initial data satisfying some compatibility conditions. The key point here is that the initial density need not be positive and may vanish in an open set.

\section{Introduction}

Compressible micropolar viscous fluids study the viscous compressible fluids with randomly oriented (or spherical) particles suspended in the medium, when the deformation of fluid particles is ignored. The theory can help us consider some physical phenomena which cannot be treated by the compressible viscous baratropic flows, due to the effect of microparticles. The microstructure of the polar fluids is mechanically significant. The governing system of equations of compressible micropolar viscous fluids expresses the balance of mass, momentum, and moment of momentum see $[1,2]$, that is,

$$
\left\{\begin{array}{l}
\rho_{t}+\operatorname{div}(\rho u)=0 \\
(\rho u)_{t}+\operatorname{div}(\rho u \otimes u)+\nabla p(\rho)=(\mu+\zeta) \Delta u+(\mu+\lambda-\zeta) \nabla \operatorname{div} u+2 \zeta \operatorname{rot} w \\
(\rho w)_{t}+\operatorname{div}(\rho u \otimes w)+4 \zeta w=\mu^{\prime} \Delta w+\left(\mu^{\prime}+\lambda^{\prime}\right) \nabla \operatorname{div} w+2 \zeta \operatorname{rot} u
\end{array}\right.
$$

Here the density $\rho=\rho(t, x)$, the velocity $u=\left[u^{1}(t, x), u^{2}(t, x), u^{3}(t, x)\right]$, the microrotational velocity $w=\left[w^{1}(t, x), w^{2}(t, x), w^{3}(t, x)\right]$, and the pressure $p(\rho)=a \rho^{\gamma}(a>0, \gamma>$ 1 ) are functions of the time $t \in(0, T)$ and the spatial coordinate $x \in \Omega$, where $\Omega$ is either a bounded open subset in $\mathbb{R}^{3}$ with smooth boundary or a usual unbounded domain such as the whole space $\mathbb{R}^{3}$, the half space $\mathbb{R}_{+}^{3}$ and an exterior domain with smooth boundary. $\mu, \lambda, \mu^{\prime}, \lambda$, and $\zeta$ are positive constants, which describe the viscosity of the fluids, satisfying the additional condition: $\mu+\lambda-\zeta \geq 0$.

We prescribe the initial boundary value conditions:

$$
\begin{aligned}
& (\rho, u, w)(0, x)=\left(\rho_{0}, u_{0}, w_{0}\right) \text { in } \Omega, \\
& (u, w)=(0,0) \quad \text { on } \quad(0, T) \times \partial \Omega, \\
& (\rho, u, w)(t, x) \rightarrow(0,0,0), \quad \text { as }|x| \rightarrow \infty,(t, x) \in(0, T) \times \Omega .
\end{aligned}
$$


There are lots of literature on the well-posedness and ill-posedness of the incompressible micropolar viscous fluid, i.e., the system (1) with $\rho \equiv$ Constant. Yamaguchi [3] considered the global strong solution in a bounded domain with small initial data. Lukaszewicz [4] showed the existence theorem for the incompressible micropolar viscous fluid with sufficiently regular initial data. And with the effect of Magnetic fields, the system of magneto-micropolar fluid, in [5], Yuan gave the local smooth solution without the smallness of the initial data; and also gave the blow-up criterion for the smooth solutions. Amirat and Hamdache [6] considered the global weak solutions with finite energy and establish the long-time behavior of the solution. And Amirat et al. [7] also proved the global in time weak solution with finite energy of an initial-boundary value problem and long-time behavior of such solution with the effect of magnetic field. We also refer the reader to [8] for local strong solution in bounded domain of $\mathbb{R}^{3}$ and references therein.

For the compressible case, in the absence of vacuum, there also have been lots of studies on the full viscous compressible micropolar fluids (which include also the conservation law of energy) since Eringen [1]. The one-dimensional problem studied by Mujaković [9-12], and Dražić and Mujaković [13] and references therein. For the multidimensional case, we refer the readers to [2,14-17] and references therein. If the vacuum occur initially, Chen $[18,19]$ studied the global strong solutions of compressible micropolar viscous fluids in 1-D. Recently, Amirat and Hamdache [20] studied the micropolar fluids with the effect of magnetic field, they prove the global weak solution in a bounded domain in $\mathbb{R}^{3}$ with initial vacuum.

Classical compressible viscous flows, i.e., $w=0$ and $\zeta=0$ in (1), have been studied by many authors. In [21], Matsumura and Nishida considered the global smooth solutions under the condition that initial data close to a non-vacuum equilibrium. For the arbitrary initial data, the major breakthrough is due to Lions [22], where he established global existence of weak solutions for the whole space, periodic domain or bounded domains with Dirichlet boundary conditions if $\gamma \geq 9 / 5$. The restriction on $\gamma$ was improved to $\gamma>3 / 2$ by Feireisl etal. in [23].

In [24], Choe and Kim established local in time strong solution of isentropic compressible fluids with initial density $\rho_{0}$ may vanish in an open subset. After that, Cho and Kim [25] studied the local existence of strong solutions of viscous polytropic fluids with vacuum. Cho et al. [26] considered the unique solvability of the initial boundary value problems for compressible viscous fluids that the initial density need not be bounded away from zero.

Hoff and Serre [27] presented an example which showed the failure of continuous dependence of weak solution, so it should be noticed that contrary to the case of strong solutions, weak solutions with vacuum need not depend continuously on their initial data.

Before stating the main result, we introduce the notions used throughout this article. We denote

$$
\int f \mathrm{~d} x=\int_{\Omega} f \mathrm{~d} x .
$$


For $1 \leq r \leq \infty$, we denote the standard homogeneous and inhomogeneous Sobolev spaces as follows:

$$
\left\{\begin{array}{l}
L^{r}=L^{r}(\Omega), D^{k, r}=\left\{u \in L_{\mathrm{loc}}^{1}(\Omega):\left|\nabla^{k} u\right| L^{r}<\infty\right\} \\
W^{k, r}=L^{r} \cap D^{k, r}, H^{k}=W^{k, 2}, D^{k}=D^{k, 2} \\
D_{0}^{1}=\left\{u \in L^{6}:|\nabla u|_{L^{2}}<\infty, \quad \text { and (3) or (4) holds }\right\} \\
H_{0}^{1}=L^{2} \cap D_{0}^{1}, \quad|u|_{D^{k, r}}=\left|\nabla^{k} u\right|_{L^{r}}
\end{array}\right.
$$

In spirit of [26], our aim of this article is to establish the local strong solution of (1)(4). Assume the initial data $\left(\rho_{0}, w_{0}, u_{0}\right)$ satisfying the regularity

$$
\rho_{0} \in H^{1} \cap W^{1, q} \text { and }\left(w_{0}, u_{0}\right) \in D_{0}^{1} \cap D^{2},
$$

where $3<q<\infty$, and the compatibilities

$$
-(\mu+\zeta) \Delta u_{0}-(\mu+\lambda-\zeta) \nabla \operatorname{div} u_{0}-2 \zeta \operatorname{rot} w_{0}+\nabla p\left(\rho_{0}\right)=\rho_{0}^{1 / 2} g_{1}
$$

and

$$
-\mu^{\prime} \Delta w_{0}-\left(\lambda^{\prime}+\mu^{\prime}\right) \nabla \operatorname{div} w_{0}+4 \zeta w_{0}-2 \zeta \operatorname{rot} u_{0}=\rho_{0}^{1 / 2} g_{2},
$$

for some $\left(g_{1}, g_{2}\right) \in L^{2}$.

Now, we state our main result in this article:

Theorem 1 Assume the data $\left(\rho_{0}, u_{0}, w_{0}\right)$ satisfy the regularity conditions (5) and the compatibility conditions (6) and (7).

Then there exists a time $T: \in(0, T)$ and a unique strong solutions $(\rho, u, w)$ to the initial boundary value problem (1)-(4) such that

$$
\begin{aligned}
& \rho \in C\left(\left[0, T_{*}\right] ; H^{1} \cap W^{1, q_{0}}\right), \quad \rho_{t} \in C\left(\left[0, T_{*}\right] ; L^{2} \cap L^{q_{0}}\right) ; \\
& (u, w) \in C\left(\left[0, T_{*}\right] ; D_{0}^{1} \cap D^{2}\right) \cap L^{2}\left(0, T_{*} ; D^{2, q_{0}}\right) ; \\
& \left(u_{t}, w_{t}\right) \in L^{2}\left(\left[0, T_{*}\right] ; D_{0}^{1}\right) \text { and }\left(\sqrt{\rho} u_{t}, \sqrt{\rho} w_{t}\right) \in L^{\infty}\left(0, T_{*} ; L^{2}\right),
\end{aligned}
$$

where $q_{0}=\min \{6, q\}$.

The article is organized as follow. In Section 2, we prove the global existence and regularity of the unique strong solutions to a linearized problem of the nonlinear problem (1)-(4). The result is used in Section 3 to construct approximate solutions to the original nonlinear problem. In Section 3, we derive some uniform bounds of the higher derivatives independent of the lower bound of the density. Moreover, we prove the convergence and obtain the local existence of strong solutions. In Section 4, we finish the proof of Theorem 1.

Remark 1 In this article, we use the following fact frequently later:

$$
\int_{\Omega} \operatorname{rot} w \cdot u \mathrm{~d} x=\int_{\Omega} \operatorname{rot} u \cdot w \mathrm{~d} x
$$

under the boundary condition of (3) or (4).

\section{Global existence for the linearized equations}

In this section, we consider the following linearized system:

$$
\left\{\begin{array}{l}
\rho_{t}+\operatorname{div}(\rho v)=0 \\
(\rho w)_{t}+\operatorname{div}(\rho v \otimes w)+4 \zeta w=\mu^{\prime} \Delta w+\left(\mu^{\prime}+\lambda^{\prime}\right) \nabla \operatorname{div} w+2 \zeta \operatorname{rot} v \\
(\rho u)_{t}+\operatorname{div}(\rho v \otimes u)+\nabla p(\rho)=(\mu+\zeta) \Delta u+(\mu+\lambda-\zeta) \nabla \operatorname{div} u+2 \zeta \operatorname{rot} w
\end{array}\right.
$$


where $v$ is a known vector fields. If the initial density $\rho_{0}$ is bounded away from zero, then we can apply standard arguments to prove the global existence of a unique strong solution to the initial boundary value problem (2)-(4) and (8), since the system can be uncoupled into a linear transport equation and two linear parabolic equations.

In this section, we prove the following existence result for the general case of nonnegative initial densities.

Theorem 2 Assume that the data $\left(\rho_{0}, u_{0}, w_{0}\right)$ satisfies the regularity conditions:

$$
0 \leq \rho_{0} \in H^{1} \cap W^{1, q}, \quad\left(u_{0}, w_{0}\right) \in D_{0}^{1} \cap D^{2},
$$

for some $3<q<\infty$ and the compatibility conditions:

$$
-\mu^{\prime} \Delta w_{0}-\left(\mu^{\prime}+\lambda^{\prime}\right) \operatorname{div} w_{0}+2 \zeta \operatorname{rot} v_{0}+4 \zeta w_{0}=\rho_{0}^{1 / 2} g_{1}
$$

and

$$
-(\mu+\zeta) \Delta u_{0}-(\mu+\lambda-\zeta) \nabla \operatorname{div} u_{0}-2 \zeta \operatorname{rot} w_{0}+\nabla p\left(\rho_{0}\right)=\rho_{0}^{1 / 2} g_{2}
$$

for some $\left(g_{1}, g_{2}\right) \in L^{2}$. If in addition, $v$ satisfies the regularity conditions:

$$
v \in L^{\infty}\left(0, T ; D_{0}^{1} \cap D^{2}\right) \cap L^{2}\left(0, T ; D^{2, q_{0}}\right) \quad \text { and } \quad v_{t} \in L^{2}\left(0, T ; D_{0}^{1}\right)
$$

where $q_{0}=\min \{6, q\}$. Then there exists a unique strong solution $(\rho, u, w)$ to the initial boundary value problems (2)-(4) and (8) such that

$$
\left\{\begin{array}{l}
\rho \in C\left([0, T] ; H^{1} \cap W^{1, q_{0}}\right), \quad \rho_{t} \in L^{\infty}\left(0, T ; L^{2} \cap L^{q_{0}}\right) \\
(u, w) \in C\left([0, T] ; D_{0}^{1} \cap D^{2}\right) \cap L^{2}\left(0, T ; D^{2, q_{0}}\right) \\
\left(u_{t}, w_{t}\right) \in L^{2}\left(0, T ; D_{0}^{1}\right), \quad\left(\sqrt{\rho} u_{t}, \sqrt{\rho} w_{t}\right) \in L^{\infty}\left(0, T ; L^{2}\right) .
\end{array}\right.
$$

Here, we emphasize that we focus on the bounded open domain $\Omega$ with smooth boundary condition. As for the unbounded domain, we can deal the same problem with the standard domain expansion technique that derived in [22]. We also refer the reader to [26]. The key of this technique is that the a priori estimates do not depend on the size of the domain. So, we here emphasize that the a priori estimates deduced in this section are independent of the size of the domain.

\subsection{Existence of theorem 2}

We begin with an existence result for the case of positive initial densities.

Lemma 1 Let $\Omega$ be a bounded domain in $\mathbb{R}^{3}$ with smooth boundary, and let $\left(\rho_{0}, u_{0}\right.$, $w_{0}$ ) be a given data satisfying the regularity condition (9)-(11). Assume further that $v_{t} \in L^{2}\left(0, T ; D_{0}^{1}\right), v_{t} \in L^{2}\left(0, T ; D_{0}^{1}\right), \rho_{0} \in H^{2}$ and $\rho_{0} \geq \delta$ in $\Omega$ for some constant $\delta>0$. Then there exists a unique strong solution $(\rho, u, w)$ to the initial boundary value problems (2), (3) and (8) such that

$$
\left\{\begin{array}{l}
\rho \in C\left([0, T] ; H^{2}\right), \quad(u, w) \in C\left([0, T] ; D_{0}^{1} \cap D^{2}\right) \cap L^{2}\left(0, T ; D^{3}\right) \\
\rho_{t} \in C\left([0, T] ; H^{1}\right), \quad\left(u_{t}, w_{t}\right) \in L^{2}\left(0, T ; D_{0}^{1}\right) \cap C\left([0, T] ; L^{2}\right) \\
\text { and } \rho>0 \text { on }[0, T] \times \bar{\Omega}
\end{array}\right.
$$


Proof Due to the classical embedding result, that $v \in C\left([0, T] ; H^{2}\right)$. Hence the existence and regularity of a unique solution of the linearized continuity Equation (8) have been well-known. Moreover, the unique solution $\rho$ can be expressed by:

$$
\rho(t, x)=\rho_{0}(U(0, t, x)) \exp \left[-\int_{0}^{t} \operatorname{div} v(s, U(s, t, x)) \mathrm{d} s\right],
$$

where $U(t, s, x)$ is the solution to

$$
\begin{cases}\frac{\partial}{\partial t} U(t, s, x)=v(t, U(t, s, x)), & 0 \leq t \leq T \\ U(s, s, x)=x, & 0 \leq s \leq T, x \in \bar{\Omega} .\end{cases}
$$

We refer the readers to [28,29] for a detailed proof. As a consequence of $(14)$ and Sobolev inequality, we have

$$
\rho(t, x) \geq \delta \exp \left[-\int_{0}^{T}|\nabla v(s)|_{L^{\infty}} \mathrm{d} s\right]>0,
$$

for $(t, x) \in[0, T] \times \bar{\Omega}$. Hence the linearized moment of momentum Equation $(8)_{2}$ can be written as a linear parabolic system

$$
w_{t}+v \cdot \nabla w+4 \rho^{-1} \zeta w-\rho^{-1} \mu^{\prime} \Delta w-\rho^{-1}\left(\mu^{\prime}+\lambda^{\prime}\right) \nabla \operatorname{div} w-2 \rho^{-1} \zeta \operatorname{rot} v=0 .
$$

The existence and regularity of the unique solution $w$ can be proved by applying classical methods, for instance, the method of continuity (see [29]). Similarly, the linearized momentum Equation $(8)_{3}$ also as a linear parabolic system

$$
u_{t}+v \cdot \nabla u-\rho^{-1}(\mu+\zeta) \Delta u-\rho^{-1}(\mu+\lambda-\zeta) \nabla \operatorname{div} u-2 \rho^{-1} \zeta \operatorname{rot} w=-\rho^{-1} \nabla p(\rho),
$$

and can be solved using the same method.

Now we prove the existence of strong solutions. Then thanks to Lemma 1, there exists a unique strong solution $(\rho, u, w)$ satisfying the regularity (13). To remove the additional hypotheses in Lemma 1 , we will derive some uniform estimates independent of $\delta,|v|_{L^{2}\left(0, T ; D^{3}\right)}$, and $\left|\rho_{0}\right|_{D^{2}}$.

First, we consider the solution $\rho$ of the linearized continuity Equation $(8)_{1}$. Since $(8)_{1}$ is a linear transport equation, so we need to prove the estimates. Multiply $(8)_{1}$ by $\rho^{r-1}$ ( $r=2$ or $q_{0}$ ) and integrating (by parts) over $\Omega$, we obtain:

$$
\frac{\mathrm{d}}{\mathrm{d} t} \int \rho^{r} \mathrm{~d} x \leq C \int|\nabla v||\rho|^{r} \mathrm{~d} x
$$

Then, using Sobolev inequality, we get

$$
\frac{\mathrm{d}}{\mathrm{d} t}|\rho|_{L^{r}}^{r} \leq C|\nabla v|_{W^{1, q_{0}}}|\rho|_{L^{r}}^{r}
$$

Differentiating $(8)_{1}$ with respect to $x_{i}$, then multiplying the resultant equation by $\partial_{i} \rho\left|\partial_{i} \rho\right|^{r-2}, i=1,2,3$ and then integrating over $\Omega$, we have

$$
\frac{\mathrm{d}}{\mathrm{d} t} \int\left|\partial_{i} \rho\right|^{r} \mathrm{~d} x \leq C \int\left(|\nabla v||\nabla \rho|^{r}+\rho|\nabla \rho|^{r-1}\left|\nabla^{2} v\right|\right) \mathrm{d} x
$$


By virtue of Sobolev inequality, we get:

$$
\frac{\mathrm{d}}{\mathrm{d} t}|\partial \rho|_{L^{r}}^{r} \leq C|\nabla v|_{H^{1} \cap D^{1, q_{0}}}|\rho|_{H^{1} \cap W^{1, q_{0}}} .
$$

Using (16) and (17) together with Gronwall's inequality, one yields:

$$
\sup _{0 \leq t \leq T}|\rho(t)|_{H^{1} \cap W^{1, q_{0}}} \leq\left|\rho_{0}\right|_{H^{1} \cap W^{1, q_{0}}} \exp \left(C \int_{0}^{T}|\nabla v(s)|_{H^{1} \cap D^{1, q_{0}}} \mathrm{~d} s\right) \leq C .
$$

Since $\rho_{t}=-v \cdot \nabla \rho-\rho \operatorname{div} v, p=p(\rho)$ and $p(0)=0$, we can easily get

$$
\sup _{0 \leq t \leq T}\left(\left|\rho_{t}\right|_{L^{2} \cap L^{q_{0}}}+|p(t)|_{H^{1} \cap W^{1, q_{0}}}+\left|p_{t}(t)\right|_{L^{2} \cap L^{q_{0}}}\right) \leq C .
$$

Then, we consider the solution $w$ of the linearized Equation $(8)_{2}$. Rewrite $(8)_{2}$, with the help of $(8)_{1}$, as:

$$
\rho w_{t}+\rho v \cdot \nabla w+4 \zeta w=\mu^{\prime} \Delta w+\left(\mu^{\prime}+\lambda^{\prime}\right) \nabla \operatorname{div} w+2 \zeta \operatorname{rot} v
$$

Multiplying this equation by $w_{t}$ and integrating (by parts) over $\Omega$, we have

$$
\begin{aligned}
& \int \rho\left|w_{t}\right|^{2} \mathrm{~d} x+\frac{\mathrm{d}}{\mathrm{d} t} \int\left(\frac{\mu^{\prime}}{2}|\nabla w|^{2}+\frac{\mu^{\prime}+\lambda^{\prime}}{2}|\operatorname{div} w|^{2}+2 \zeta|w|^{2}\right) \mathrm{d} x \\
= & -\int\left(\rho v \cdot \nabla w w_{t}+2 \zeta \operatorname{rot} v w_{t}\right) \mathrm{d} x \\
= & -\int\left(\rho v \cdot \nabla w w_{t}+2 \zeta \operatorname{rot} v_{t} w\right) \mathrm{d} x+\frac{\mathrm{d}}{\mathrm{d} t} \int 2 \zeta \operatorname{rot} v w \mathrm{~d} x .
\end{aligned}
$$

Then, using Young's inequality, we obtain:

$$
\begin{aligned}
& \frac{1}{2} \int \rho\left|w_{t}\right|^{2} \mathrm{~d} x+\frac{d}{\mathrm{~d} t} \int\left(\frac{\mu^{\prime}}{2}|\nabla w|^{2}+\frac{\mu^{\prime}+\lambda^{\prime}}{2}|\operatorname{div} w|^{2}+2 \zeta|w|^{2}\right) \mathrm{d} x \\
\leq & \int\left(\rho|v|^{2}|\nabla w|^{2}+\zeta\left|\nabla v_{t}\right|^{2}+\zeta|w|^{2}\right) \mathrm{d} x+\frac{\mathrm{d}}{\mathrm{d} t} \int 2 \zeta \operatorname{rot} v w \mathrm{~d} x .
\end{aligned}
$$

Integrating the above inequality over $(0, t)$, we get:

$$
\begin{aligned}
& \int_{0}^{t}\left|\sqrt{\rho} w_{t}\right|_{L^{2}}^{2} \mathrm{~d} s+|\nabla w|_{L^{2}}^{2}+2 \zeta|w|_{L^{2}}^{2} \\
& \leq C+C \int_{0}^{t}|w|_{L^{2}}^{2} \mathrm{~d} s+\int_{0}^{t} \int \rho|v|^{2}|\nabla w|^{2} \mathrm{~d} x \mathrm{~d} s+2 \zeta \int|\operatorname{rot} v||w| \mathrm{d} x \\
& \leq C+C \int_{0}^{t}\left(|w|_{L^{2}}^{2}+|\nabla w|_{L^{2}}^{2}\right) \mathrm{d} s+\zeta|\nabla v|_{L^{2}}^{2}+\zeta|w|_{L^{2}}^{2} .
\end{aligned}
$$

Thus, we have

$$
\int_{0}^{t}\left|\sqrt{\rho} w_{t}\right|_{L^{2}}^{2} \mathrm{~d} s+|\nabla w|_{L^{2}}^{2}+|w|_{L^{2}}^{2} \leq C \int_{0}^{t}\left(|\nabla w|_{L^{2}}^{2}+|w|_{L^{2}}^{2}\right) \mathrm{d} s+C .
$$

Therefore, in view of Gronwall's inequality, we have:

$$
\int_{0}^{T}\left|\sqrt{\rho} w_{t}\right|_{L^{2}}^{2} \mathrm{~d} s+\sup _{0 \leq t \leq T}\left(|\nabla w|_{L^{2}}^{2}+|w|_{L^{2}}^{2}\right) \leq C
$$


To derive higher regularity estimates, we differentiate (19) with respect to $t$ and obtain:

$$
\begin{aligned}
& \rho w_{t t}+\rho v \cdot \nabla w_{t}+4 \zeta w_{t}-\mu^{\prime} \Delta w_{t}-\left(\mu^{\prime}+\lambda^{\prime}\right) \nabla \operatorname{div} w_{t} \\
= & -\rho_{t} w_{t}-\rho_{t} v \cdot \nabla w-\rho v_{t} \cdot \nabla w+2 \zeta \operatorname{rot} v_{t}
\end{aligned}
$$

Multiplying the above equation by $w_{t}$, and integrating over $\Omega$, we have:

$$
\begin{aligned}
& \frac{1}{2} \frac{\mathrm{d}}{\mathrm{d} t}\left|\sqrt{\rho} w_{t}\right|_{L^{2}}^{2}+4 \zeta\left|w_{t}\right|_{L^{2}}^{2}+\mu^{\prime}\left|\nabla w_{t}\right|_{L^{2}}^{2} \\
\leq & \int\left(\rho|v|\left|\nabla w_{t}\right|\left|w_{t}\right|+\left|\rho_{t}\right||v||\nabla w|\left|w_{t}\right|+\rho\left|v_{t}\right||\nabla w|\left|w_{t}\right|+2 \zeta\left|\nabla v_{t}\right|\left|w_{t}\right| \mathrm{d} x=\sum_{i=1}^{4} I_{i} .\right)
\end{aligned}
$$

Now, we estimate each term on the right-hand side of (24)

$$
\begin{aligned}
I_{1} & =\int \rho|v|\left|\nabla w_{t}\right|\left|w_{t}\right| \mathrm{d} x \leq|\rho|_{L^{\infty}}^{1 / 2}|v|_{L^{\infty}}\left|\sqrt{\rho} w_{t}\right|_{L^{2}}\left|\nabla w_{t}\right|_{L^{2}} \\
& \leq C|\rho|_{L^{\infty}}|v|_{L^{\infty}}^{2}\left|\sqrt{\rho} w_{t}\right|_{L^{2}}^{2}+\varepsilon\left|\nabla w_{t}\right|_{L^{2}}^{2} ; \\
I_{2} & =\int\left|\rho_{t}\right||v||\nabla w|\left|w_{t}\right| \mathrm{d} x \leq|v|_{L^{\infty}}\left|\rho_{t}\right|_{L^{3}}|\nabla w|_{L^{2}}\left|w_{t}\right|_{L^{6}} \\
& \leq C|v|_{L^{\infty}}\left|\rho_{t}\right|_{L^{2}}^{\alpha}\left|\rho_{t}\right|_{L^{q_{0}}}^{1-\alpha}|\nabla w|_{L^{2}}\left|\nabla w_{t}\right|_{L^{2}} \\
& \leq C|v|_{L^{\infty}}^{2}\left|\rho_{t}\right|_{L^{2}}^{2 \alpha}\left|\rho_{t}\right|_{L^{q_{0}}}^{2(1-\alpha)}|\nabla w|_{L^{2}}^{2}+\varepsilon\left|\nabla w_{t}\right|_{L^{2}}^{2} ; \\
I_{3} & =\int \rho\left|v_{t}\right||\nabla w|\left|w_{t}\right| \mathrm{d} x \leq|\rho|_{L^{\infty}}^{1 / 2}\left|v_{t}\right|_{L^{6}}|\nabla w|_{L^{2}}\left|\sqrt{\rho} w_{t}\right|_{L^{3}} \\
& \leq|\rho|_{L^{\infty}}^{1 / 2}\left|\nabla v_{t}\right|_{L^{2}}|\nabla w|_{L^{2}}\left|\sqrt{\rho} w_{t}\right|_{L^{2}}^{1 / 2}\left|\sqrt{\rho} w_{t}\right|_{L^{6}}^{1 / 2} \\
& \leq C|\rho|_{L^{\infty}}^{3 / 4}\left|\nabla v_{t}\right|_{L^{2}}|\nabla w|_{L^{2}}\left|\sqrt{\rho} w_{t}\right|_{L^{2}}^{1 / 2}\left|\nabla w_{t}\right|_{L^{2}}^{1 / 2} \\
& \leq C|\rho|_{L^{\infty}}^{3}|\nabla w|_{L^{2}}^{4}\left|\sqrt{\rho} w_{t}\right|_{L^{2}}^{4}+C\left|\nabla v_{t}\right|_{L^{2}}^{2}+\varepsilon\left|\nabla w_{t}\right|_{L^{2}}^{2} ; \\
I_{4} & =2 \zeta \int\left|\nabla v_{t}\right|\left|w_{t}\right| \mathrm{d} x \leq \zeta\left|\nabla v_{t}\right|_{L^{2}}^{2}+\zeta\left|w_{t}\right|_{L^{2}}^{2} .
\end{aligned}
$$

Choosing $\varepsilon=\mu^{\prime} / 6$, substitute the above estimates into (24), we can get

$$
\frac{\mathrm{d}}{\mathrm{d} t}\left|\sqrt{\rho} w_{t}\right|_{L^{2}}^{2}+\frac{\mu^{\prime}}{2}\left|\nabla w_{t}\right|_{L^{2}}^{2}+2 \zeta\left|w_{t}\right|_{L^{2}}^{2} \leq C\left(1+\left|\nabla v_{t}\right|_{L^{2}}^{2}+\left|\sqrt{\rho} w_{t}\right|_{L^{2}}^{2}\right)
$$

Integrating over $(\tau, t)$ for some fixed $\tau \in(0, T)$, we deduce that

$$
\left|\sqrt{\rho} w_{t}\right|_{L^{2}}^{2}+\int_{\tau}^{T}\left(\left|\nabla w_{t}\right|_{L^{2}}^{2}+2 \zeta\left|w_{t}\right|_{L^{2}}^{2}\right) \mathrm{d} s \leq C+C\left|\sqrt{\rho} w_{t}(\tau)\right|_{L^{2}}^{2} \quad \text { for } \quad \tau \leq t \leq T .(26)
$$

To estimate $\left|\sqrt{\rho} w_{t}(\tau)\right|_{L^{2}}^{2}$, due to(19), we see

$$
\int \rho\left|w_{t}\right|^{2} \mathrm{~d} x \leq 4 \int \rho|v|^{2}|\nabla w|^{2} \mathrm{~d} x+\int \rho^{-1}\left|-\mu^{\prime} \Delta w-\left(\mu^{\prime}+\lambda^{\prime}\right) \nabla \operatorname{div} w-2 \zeta \operatorname{rot} v+4 \zeta w\right|^{2} \mathrm{~d} x,
$$

and thus we have

$$
\limsup _{\tau \rightarrow 0}\left|\sqrt{\rho} w_{t}(\tau)\right|_{L^{2}}^{2} \leq C\left(1+C_{1}\left(\rho_{0}, u_{0}, w_{0}\right)\right),
$$


where $\mathcal{C}_{1}\left(\rho_{0}, u_{0}, w_{0}\right)$ is defined by

$$
\mathcal{C}_{1}\left(\rho_{0}, u_{0}, w_{0}\right)=\int \rho_{0}^{-1}\left|-\mu^{\prime} \Delta w_{0}-\left(\mu^{\prime}+\lambda^{\prime}\right) \nabla \operatorname{div} w_{0}-2 \zeta \operatorname{rot} v_{0}+4 \zeta w_{0}\right|^{2} \mathrm{~d} x
$$

Therefore, letting $\tau \rightarrow 0$ in (26), we conclude that

$$
\sup _{0 \leq t \leq T}\left|\sqrt{\rho} w_{t}(t)\right|_{L^{2}}^{2}+\int_{0}^{T}\left(\left|\nabla w_{t}\right|_{L^{2}}^{2}+\left|w_{t}\right|_{L^{2}}^{2}\right) \mathrm{d} t \leq C\left(1+\mathcal{C}_{1}\left(\rho_{0}, u_{0}, w_{0}\right)\right) .
$$

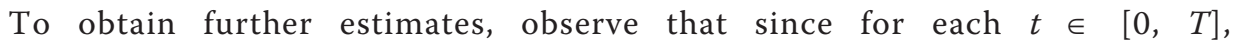
$w=w(t) \in D_{0}^{1} \cap D^{2}$ is a solution of the elliptic system:

$$
\mu^{\prime} \Delta w+\left(\mu^{\prime}+\lambda^{\prime}\right) \nabla \operatorname{div} w-4 \zeta w=F_{1},
$$

where $F_{1}=-\rho w_{t}-\rho v \cdot w+2 \zeta \operatorname{rot} v \in L^{2} \cap L^{q_{0}}$. And then

$$
|w|_{D^{2, r}} \leq C\left|-\rho w_{t}-\rho v \cdot w+2 \zeta \operatorname{rot} v\right|_{L^{r}}+C|w|_{D^{1, r}}, r=2, q_{0} .
$$

Therefore, using the previous estimates, we can deduce from (28) that

$$
\sup _{0 \leq t \leq T}|w(t)|_{D^{2}}^{2}+\int_{0}^{T}|w(t)|_{D^{2, q_{0}}}^{2} \mathrm{~d} t \leq C\left(1+\mathcal{C}_{1}\left(\rho_{0}, u_{0}, w_{0}\right)\right) .
$$

Now, we consider the solution $u$ of the linearized Equation $(8)_{3}$. Rewrite $(8)_{3}$ as

$$
\rho u_{t}+\rho v \cdot \nabla u+\nabla p(\rho)=(\mu+\zeta) \Delta u+(\mu+\lambda-\zeta) \nabla \operatorname{div} u+2 \zeta \operatorname{rot} w .
$$

Multiplying this equation by $u_{t}$ and integrating over $\Omega$, we have

$$
\begin{aligned}
& \int \rho\left|u_{t}\right|^{2} \mathrm{~d} x+\frac{\mathrm{d}}{\mathrm{d} t} \int\left(\frac{\mu+\zeta}{2}|\nabla u|^{2}+\frac{\mu+\lambda-\zeta}{2}|\operatorname{div} u|^{2}-p(\rho)-2 \zeta \operatorname{rot} w u\right) \mathrm{d} x \\
= & \int\left(\rho v \cdot \nabla u u_{t}-p(\rho)_{t} \operatorname{div} u-2 \zeta \operatorname{rot} w_{t} u\right) \mathrm{d} x .
\end{aligned}
$$

Then, using Young's inequality, we obtain:

$$
\begin{aligned}
& \frac{1}{2} \int \rho\left|u_{t}\right|^{2} \mathrm{~d} x+\frac{\mathrm{d}}{\mathrm{d} t} \int\left(\frac{\mu+\zeta}{2}|\nabla u|^{2}+\frac{\mu+\lambda-\zeta}{2}|\operatorname{div} u|^{2}-p(\rho)-2 \zeta \operatorname{rot} w u\right) \mathrm{d} x \\
\leq & \int\left(\frac{1}{2} \rho|v|^{2}|\nabla u|^{2}+\left|p(\rho)_{t}\right||\nabla u|+\zeta\left|w_{t}\right|^{2}+\zeta|\nabla u|^{2}\right) \mathrm{d} x .
\end{aligned}
$$

Integrating the above inequality over $(0, t)$ using Young's inequality, we get

$$
\int_{0}^{t}\left|\sqrt{\rho} u_{t}\right|_{L^{2}}^{2} \mathrm{~d} s+|\nabla u|_{L^{2}}^{2} \leq C\left(1+\int_{0}^{t}|\nabla u|_{L^{2}}^{2} \mathrm{~d} s\right) .
$$

Therefore, in view of Gronwall's inequality, we have

$$
\int_{0}^{T}\left|\sqrt{\rho} u_{t}\right|_{L^{2}}^{2} \mathrm{~d} t+\sup _{0 \leq t \leq T}|u(t)|_{D_{0}^{1}}^{2} \leq C .
$$

To derive higher regularity estimates, differentiate (30) with respect to $t$ and obtain:

$$
\begin{aligned}
& \rho u_{t t}+\rho v \cdot \nabla u_{t}+\nabla p(\rho)_{t}-(\mu+\zeta) \Delta u_{t}-(\mu+\lambda-\zeta) \nabla \operatorname{div} u_{t} \\
= & -\rho_{t} u_{t}-\rho_{t} v \cdot \nabla u-\rho v_{t} \cdot u+2 \zeta \operatorname{rot} w_{t} .
\end{aligned}
$$


Multiplying the above equation by $u_{t}$, integrating (by parts) over $\Omega$, and using $(8)_{1}$, we obtain:

$$
\begin{aligned}
& \frac{1}{2} \frac{d}{d t} \int \rho\left|u_{t}\right|^{2} \mathrm{~d} x+\int\left((u+\zeta)\left|\nabla u_{t}\right|^{2}+(\mu+\lambda-\zeta)\left|\operatorname{div} u_{t}\right|^{2}\right) \mathrm{d} x \\
\leq & \int \rho|v|\left|\nabla u_{t}\right|\left|u_{t}\right| \mathrm{d} x+\int\left|\rho_{t}\right||v||\nabla u|\left|u_{t}\right| \mathrm{d} x+\int \rho\left|v_{t}\right||\nabla u|\left|u_{t}\right| \mathrm{d} x \\
+ & \int\left|p(\rho)_{t}\right|\left|\nabla u_{t}\right| \mathrm{d} x+2 \zeta \int\left|w_{t}\right|\left|\nabla u_{t}\right| \mathrm{d} x=\sum_{i=1}^{5} I I_{i} .
\end{aligned}
$$

Using Sobolev inequality, interpolation inequality and Young's inequality, we obtain:

$$
\begin{aligned}
& I I_{1}=\int \rho|v|\left|\nabla u_{t}\right|\left|u_{t}\right| \mathrm{d} x \leq|\rho|_{L^{\infty}}^{1 / 2}|v|_{L^{\infty}}\left|\sqrt{\rho} u_{t}\right|_{L^{2}}\left|\nabla u_{t}\right|_{L^{2}} \\
& \leq C|\rho|_{L^{\infty}}|v|_{L^{\infty}}^{2}\left|\sqrt{\rho} u_{t}\right|_{L^{2}}^{2}+\varepsilon\left|\nabla u_{t}\right|_{L^{2}}^{2} \\
& I I_{2}=\int\left|\rho_{t}\right||v||\nabla u|\left|u_{t}\right| \mathrm{d} x \leq|v|_{L^{\infty}}\left|\rho_{t}\right|_{L^{3}}|\nabla u|_{L^{2}}\left|u_{t}\right|_{L^{6}} \\
& \leq|v|_{L^{\infty}}\left|\rho_{t}\right|_{L^{2}}^{\alpha}\left|\rho_{t}\right|_{L^{q_{0}}}^{1-\alpha}|\nabla u|_{L^{2}}\left|\nabla u_{t}\right|_{L^{2}} \\
& \leq C|v|_{L^{\infty}}^{2}\left|\rho_{t}\right|_{L^{2}}^{2 \alpha}\left|\rho_{t}\right|_{L^{q_{0}}}^{2(1-\alpha)}|\nabla u|_{L^{2}}^{2}+\varepsilon\left|\nabla u_{t}\right|_{L^{2}}^{2} ; \\
& I I_{3}=\int \rho\left|v_{t}\right||\nabla u|\left|u_{t}\right| \mathrm{d} x \leq|\rho|_{L^{\infty}}^{1 / 2}\left|u_{t}\right|_{L^{6}}|\nabla u|_{L^{2}}\left|\sqrt{\rho} u_{t}\right|_{L^{3}} \\
& \leq C|\rho|_{L^{\infty}}^{1 / 2}\left|\nabla v_{t}\right|_{L^{2}}|\nabla u|_{L^{2}}\left|\sqrt{\rho} u_{t}\right|_{L^{2}}^{1 / 2}\left|\sqrt{\rho} u_{t}\right|_{L^{6}}^{1 / 2} \\
& \leq C|\rho|_{L^{\infty}}^{3 / 4}\left|\nabla v_{t}\right|_{L^{2}}|\nabla u|_{L^{2}}\left|\sqrt{\rho} u_{t}\right|_{L^{2}}^{1 / 2}\left|\nabla u_{t}\right|_{L^{2}}^{1 / 2} \\
& \leq C|\rho|_{L^{\infty}}^{3}|\nabla u|_{L^{2}}^{4}\left|\sqrt{\rho} u_{t}\right|_{L^{2}}^{2}+C\left|\nabla v_{t}\right|_{L^{2}}^{2}+\varepsilon\left|\nabla u_{t}\right|_{L^{2}}^{2} \\
& I I_{4}=\int\left|p(\rho)_{t}\right|\left|\nabla u_{t}\right| \mathrm{d} x \leq C\left|p(\rho)_{t}\right|_{L^{2}}^{2}+\varepsilon\left|\nabla u_{t}\right|_{L^{2}}^{2} \\
& I I_{5}=2 \zeta \int\left|w_{t}\right|\left|\nabla u_{t}\right| \mathrm{d} x \leq \zeta\left|w_{t}\right|_{L^{2}}^{2}+\zeta\left|\nabla u_{t}\right|_{L^{2}}^{2}
\end{aligned}
$$

Substituting the above estimates into (32), we can easily show that:

$$
\frac{\mathrm{d}}{\mathrm{d} t}\left|\sqrt{\rho} u_{t}\right|_{L^{2}}^{2}+\left|\nabla u_{t}\right|_{L^{2}}^{2} \leq C\left(1+\left|\nabla v_{t}\right|_{L^{2}}^{2}+\left|\sqrt{\rho} u_{t}\right|_{L^{2}}^{2}\right) .
$$

Now, for fixed $\tau \in(0, T)$. Since the right-hand side of (33) is integrable in $(0, T)$, we deduce that

$$
\left|\sqrt{\rho} u_{t}(t)\right|_{L^{2}}^{2}+\int_{\tau}^{t}\left|\nabla u_{t}\right|_{L^{2}}^{2} \mathrm{~d} s \leq C+C\left|\sqrt{\rho} u_{t}(\tau)\right|_{L^{2}}^{2} \text { for } \tau \leq t \leq T .
$$

To estimate $\left|\sqrt{\rho} u_{t}(\tau)\right|_{L^{2}}^{2}$ from $(8)_{3}$, we see that

$$
\left|\sqrt{\rho} u_{t}(\tau)\right|_{L^{2}}^{2} \leq 4 \int \rho|v|^{2}|\nabla u|^{2} \mathrm{~d} x \int \rho^{-1}|-(\mu+\lambda) \Delta u-(\mu+\lambda-\zeta) \Delta \operatorname{div} u-2 \zeta \operatorname{rot} w+\nabla p(\rho)|^{2} \mathrm{~d} x
$$

and thus

$$
\limsup _{\tau \rightarrow 0}\left|\sqrt{\rho} u_{t}(\tau)\right|_{L^{2}}^{2} \leq C\left(1+\mathcal{C}_{2}\left(\rho_{0}, u_{0}, w_{0}\right)\right)
$$


where $\mathcal{C}_{2}\left(\rho_{0}, u_{0}, w_{0}\right)$ is defined by

$$
\mathcal{C}_{2}\left(\rho_{0}, u_{0}, w_{0}\right)=\int \rho_{0}^{-1}\left|-(\mu+\lambda) \Delta u_{0}-(\mu+\lambda-\zeta) \nabla \operatorname{div} u_{0}-2 \zeta \operatorname{rot} w_{0}+\nabla p\left(\rho_{0}\right)\right|^{2} \mathrm{~d} x
$$

Therefore, letting $\tau \rightarrow 0$ in (34), we conclude that

$$
\sup _{0 \leq t \leq T}\left|\sqrt{\rho} u_{t}\right|_{L^{2}}^{2}+\int_{0}^{T}\left|\nabla u_{t}\right|_{L^{2}}^{2} \mathrm{~d} t \leq C\left(1+\mathcal{C}_{2}\left(\rho_{0}, u_{0}, w_{0}\right)\right) .
$$

To obtain further estimates, observe that for each $t \in[0, T], u=u(t) \in D_{0}^{1} \cap D^{2}$ is a solution of the following elliptic system:

$$
-(\mu+\lambda) \Delta u-(\mu+\lambda-\zeta) \nabla \operatorname{div} u=F_{2}
$$

where $F_{2}=-\rho u_{t}-\rho v \cdot \nabla u+2 \zeta \operatorname{rot} w-\nabla p(\rho) \in L^{2} \cap L^{q_{0}}$. It follows from the elliptic regularity theory, we get

$$
|u|_{D^{2, r}} \leq C\left|-\rho u_{t}-\rho v \cdot \nabla u+2 \zeta \operatorname{rot} w-\nabla p(\rho)\right|_{L^{r}}+C|u|_{D^{1, r}}, \quad r=2, q_{0} .
$$

Therefore, using the previous estimates, we get from (36) that

$$
\sup _{0 \leq t \leq T}|u(t)|_{D^{2}}^{2}+\int_{0}^{T}|u(t)|_{D^{2, q_{0}}}^{2} \mathrm{~d} t \leq C\left(1+\mathcal{C}_{2}\left(\rho_{0}, u_{0}, w_{0}\right)\right)
$$

Since the estimates we have derived, we prove the existence result. First, we consider for the case of bounded domain. Using standard regularization techniques, we choose $p^{\delta}=p^{\delta}(\cdot)$ and $v^{\delta}, 0<\delta \ll 1$, so that

$$
\begin{aligned}
& p^{\delta}(\cdot) \in C^{2}[0, \infty), p^{\delta} \rightarrow p \text { in } C^{1}[0, \infty) \\
& v^{\delta} \in L^{\infty}\left(0, T ; D_{0}^{1} \cap D^{2}\right) \cap L^{2}\left(0, T ; D^{3}\right), v_{t}^{\delta} \in L^{2}\left(0, T ; D_{0}^{1}\right), \\
& \left(v^{\delta}, v_{t}^{\delta}\right) \rightarrow\left(v, v_{t}\right) \text { in } L^{\infty}\left(0, T ; D_{0}^{1} \cap D^{2}\right) \cap L^{2}\left(0, T ; D^{2, q_{0}}\right) \times L^{2}\left(0, T ; D_{0}^{1}\right) .
\end{aligned}
$$

Then, for each $\delta \in(0,1)$, let $\rho_{0}^{\delta}=\rho_{0}+\delta$ and let $\left(u_{0}^{\delta}, w_{0}^{\delta}\right) \in D_{0}^{1} \cap D^{2}$ is the solution to the boundary value problem:

$$
\left\{\begin{array}{l}
-(\mu+\zeta) \Delta u_{0}^{\delta}-(\mu+\lambda-\zeta) \nabla \operatorname{div} u_{0}^{\delta}-2 \zeta \operatorname{rot} w_{0}^{\delta}=-\nabla p^{\delta}\left(\rho_{0}^{\delta}\right)+\left(\rho_{0}^{\delta}\right)^{1 / 2} g_{1} \\
-\mu^{\prime} \Delta w_{0}^{\delta}-\left(\mu^{\prime}+\lambda^{\prime}\right) \nabla \operatorname{div} w_{0}^{\delta}+4 \zeta w_{0}^{\delta}=\left(\rho_{0}^{\delta}\right)^{1 / 2} g_{2} \\
\left(u_{0}^{\delta}, w_{0}^{\delta}\right)=0, \quad \text { on } \partial \Omega
\end{array}\right.
$$

It follows from the elliptic regularity result that $\left(u_{0}^{\delta}, w_{0}^{\delta}\right) \rightarrow\left(u_{0}, w_{0}\right)$ in $D_{0}^{1} \cap D^{2}$ as $\delta$ $\rightarrow 0$. Hence, if we denote by $\left(\rho^{\delta}, u^{\delta}, w^{\delta}\right)$ the solution of (8) with the initial data $\left(\rho_{0}^{\delta}, u_{0}^{\delta}, w_{0}^{\delta}\right)$ and $(p, v)$ replaced by $\left(p^{\delta}, v^{\delta}\right)$, it satisfies the estimates (35), (37), (31), (23), (18), (27), (29), where $\mathcal{C}_{1}\left(\rho_{0}, u_{0}, w_{0}\right)=\left|g_{1}\right|_{L^{2}}^{2} \leq C, \mathcal{C}_{2}\left(\rho_{0}, u_{0}, w_{0}\right)=\left|g_{2}\right|_{L^{2}}^{2} \leq C$. Therefore, we conclude that a subsequence of solutions $\left(\rho^{\delta}, u^{\delta}, w^{\delta}\right)$ converges to a limit ( $\rho$, $u, w)$ in a weak sense. Then it can easy to show that $(\rho, u, w)$ is a weak solution to the original problem (8). Moreover, due to the lower semi-continuity of various norms, we have the following regularity estimates for $(\rho, u, w)$ :

$$
\begin{aligned}
& \underset{0 \leq t \leq T}{\operatorname{ess} \sup _{0}}\left(|\rho|_{H^{1} \cap W^{1, q_{0}}}+\left|\rho_{t}\right|_{L^{2} \cap L^{q_{0}}}+|(u, w)|_{D_{0}^{1} \cap D^{2}}+\left|\left(\sqrt{\rho} u_{t}, \sqrt{\rho} w_{t}\right)\right|_{L^{2}}\right) \\
& +\int_{0}^{T}\left(|(u, w)|_{D^{2, q_{0}}}^{2}+\left|\left(u_{t}, w_{t}\right)\right|_{D_{0}^{1}}\right) \mathrm{d} t \leq C .
\end{aligned}
$$




\subsection{Continuity and uniqueness}

Now, turn our attention to the continuity of the solution $(\rho, u, w)$. The continuity of $\rho$ can be proved by a standard argument from the theory of hyperbolic equations. Since $\rho$ satisfies the regularity (38), it follows from a result of DiPerna and Lions [30] and classical embedding results that (See [31]):

$$
\rho \in C\left([0, T] ; L^{2} \cap L^{q_{0}}\right) \cap C\left([0, T] ; H^{1} \cap W^{1, q_{0}}-\text { weak }\right) .
$$

To show the strong continuity in $H^{1} \cap W^{1, q_{0}}$, observe from (17), (18) and $i=1,2,3$,

$$
\left|\partial_{i} \rho(t)\right|_{L^{r}}^{r} \leq\left|\partial_{i} \rho(0)\right|_{L^{r}}^{r}+C \int_{0}^{t}|\nabla v(s)|_{H^{1} \cap D^{1, q_{0}}} \mathrm{~d} s
$$

and thus $\limsup _{t \rightarrow 0^{+}}\left|\partial_{i} \rho(t)\right|_{L^{r}}^{r} \leq\left|\partial_{i} \rho(0)\right|_{L^{r}}^{r}$. Hence it follows from a well-known criterion on the strong conver-gence for the space $L^{r}$ (See [32]) that

$$
\lim _{t \rightarrow 0^{+}}\left|\partial_{i} \rho(t)-\partial_{i} \rho(0)\right|_{L^{r}}^{r}=0
$$

Therefore, the continuity of $\nabla \rho$ in $L^{r}\left(r=2, q_{0}\right)$ follows from the result and the observation that for each fixed $t_{0} \in[0, t]$, the function $\tilde{\rho}=\tilde{\rho}(t, x)=\tilde{\rho}\left( \pm t+t_{0}, x\right)$ is a unique strong solution to the similar initial value problem:

$$
\tilde{\rho}_{t}+\operatorname{div}(\tilde{\rho} \tilde{v})=0, \quad \text { and } \quad \tilde{\rho}(0)=\rho\left(t_{0}\right),
$$

where $\tilde{v}=\tilde{v}(t, x)= \pm v\left( \pm t+t_{0}, x\right)$.

To show the continuity of $w$, we first observe that

$$
w, v \in C\left([0, T] ; D_{0}^{1}\right) \cap C\left([0, T] ; D^{2}-\text { weak }\right) .
$$

We now prove the continuity of $\rho w_{t}$ in $L^{2}$. For a.e. $t \in(0, T)$ and $\varphi \in H_{0}^{1}$ the from (19), we have

$$
\begin{aligned}
\left(\rho w_{t}, \varphi\right)_{L^{2}} & =\left(-\rho v \cdot \nabla w-4 \zeta w+\mu^{\prime} \nabla w+\left(\mu^{\prime}+\lambda^{\prime}\right) \nabla \operatorname{div} w+2 \zeta \operatorname{rot} v, \varphi\right)_{L^{2}} \\
& =(-\rho v \cdot \nabla w-4 \zeta w+2 \zeta \operatorname{rot} v, \varphi)_{L^{2}}-\mu^{\prime}(\nabla w, \nabla \varphi)_{L^{2}}-\left(\mu^{\prime}+\lambda^{\prime}\right)(\operatorname{div} w, \operatorname{div} \varphi)_{L^{2}}
\end{aligned}
$$

and thus

$$
\frac{\mathrm{d}}{\mathrm{d} t}\left(\rho w_{t}, \varphi\right)_{L^{2}}=\left((-\rho v \cdot \nabla w-4 \zeta w+2 \zeta \operatorname{rot} v)_{t}, \varphi\right)_{L^{2}}-\mu^{\prime}\left(\nabla w_{t}, \nabla \varphi\right)_{L^{2}}-\left(\mu^{\prime}+\lambda^{\prime}\right)\left(\operatorname{div} w_{t}, \operatorname{div} \varphi\right)_{L^{2}}
$$

Using the regularity (38) of $(\rho, w)$, we show that the right-hand side of (39) is bounded above by $A_{1}(t)|\varphi|_{H_{0}^{1}}$ for some positive function $A_{1}(t) \in L^{2}(0, T)$. Hence it follows, from the well-known result (see [31]) that $\left(\rho w_{t}\right)_{t} \in L^{2}\left(0, T ; H^{-1}\right)$ and $\frac{\mathrm{d}}{\mathrm{d} t}\left(\rho w_{t}, \varphi\right)_{L^{2}}=\left\langle\left(\rho w_{t}\right)_{t}, \varphi\right\rangle$ for all $\varphi \in H_{0}^{1}$ where $\langle\cdot, \cdot\rangle$ denotes the dual pairing of $H^{-1}$ and $H_{0}^{1}$. Then since $\rho w_{t} \in L^{2}\left(0, T ; H_{0}^{1}\right)$, it follows from a standard embedding result $\rho w_{t} \in$ $C\left([0, T] ; L^{2}\right)$. Therefore, we conclude that for each $t \in[0, T], w=w(t, x) \in D_{0}^{1} \cap D^{2}$ is a solution of the elliptic system:

$$
-\mu^{\prime} \Delta w-\left(\mu^{\prime}+\lambda^{\prime}\right) \nabla \operatorname{div} w=G_{1}-\rho v \cdot \nabla w
$$


where $G_{1}=-\rho w_{t}-4 \zeta w \in C\left([0, T] ; L^{2}\right)$. Now we turn to show that $w \in C\left([0, T] ; D^{2}\right)$. In view of the elliptic regularity estimate (36), we obtain

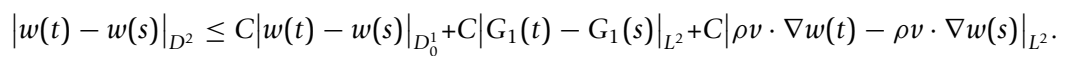

Using the estimate (36), we obtain:

$$
\begin{aligned}
& C|\rho v \cdot \nabla w(t)-\rho v \cdot \nabla w(s)|_{L^{2}} \\
\leq & C|(\rho(t)-\rho(s)) v(t) \cdot \nabla w(t)|_{L^{2}}+C|\rho(s)(v(t)-v(s)) \cdot \nabla w(t)|_{L^{2}} \\
& +C|\rho(s) v(s) \cdot(\nabla w(t)-\nabla w(s))|_{L^{2}} \\
\leq & C|\rho(t)-\rho(s)|_{L^{\infty}}|v(t)|_{L^{6}}|\nabla w(t)|_{L^{3}}+C|\rho(s)|_{L^{\infty}}|v(t)-v(s)|_{L^{6}}|\nabla w(t)|_{L^{3}} \\
& \left.+C|\rho(s)|_{L^{\infty}}|v(s)|_{L^{6}} \nabla w(t)-\nabla w(s)\right)_{L^{3}} \\
\leq & C|\rho(t)-\rho(s)|_{L^{\infty}}|\nabla v(t)|_{L^{2}}|\nabla w(t)|_{L^{2}}^{1 / 2}\left|\nabla^{2} w(t)\right|_{L^{2}}^{1 / 2} \\
& +C|\rho(s)|_{L^{\infty}}|\nabla v(t)-\nabla v(s)|_{L^{2}}|\nabla w(t)|_{L^{2}}^{1 / 2}\left|\nabla^{2} w(t)\right|_{L^{2}}^{1 / 2} \\
& +C|\rho(s)|_{L^{\infty}}|\nabla v(s)|_{L^{2}}|\nabla w(t)-\nabla w(s)|_{L^{2}}^{1 / 2}\left|\nabla^{2} w(t)-\nabla^{2} w(s)\right|_{L^{2}}^{1 / 2} \\
\leq & C\left(|\rho(t)-\rho(s)|_{L^{\infty}}+|\nabla v(t)-\nabla v(s)|_{L^{2}}+|\nabla w(t)-\nabla w(s)|_{L^{2}}\right) \\
& +\frac{1}{2}|w(t)-w(s)|_{D^{2}} .
\end{aligned}
$$

Substituting this into (40), we conclude that

$$
|w(t)-w(s)|_{D^{2}} \rightarrow 0 \text { as } t \rightarrow s .
$$

The continuity of $\rho u_{t}$ in $L^{2}$ is similar as the proof of $\rho w_{t}$. From (30), for a.e. $t \in(0$, $T)$, and all $\psi \in H_{0}^{1}$, we have

$$
\begin{aligned}
\left(\rho u_{t}, \psi\right)_{L^{2}} & =(-\rho u \cdot \nabla u+(\mu+\lambda) \Delta u+(\mu+\lambda-\zeta) \nabla \operatorname{div} u+2 \zeta \operatorname{rot} w-\nabla p(\rho), \psi)_{L^{2}} \\
& =(-\rho u \cdot \nabla u+2 \zeta \operatorname{rot} w, \psi)_{L^{2}}-((\mu+\zeta) \nabla u, \nabla \psi)_{L^{2}}-((\mu+\lambda-\zeta) \nabla \operatorname{div} u-p(\rho), \operatorname{div} \psi)_{L^{2}}
\end{aligned}
$$

and thus

$$
\begin{aligned}
\frac{\mathrm{d}}{\mathrm{d} t}\left(\rho u_{t}, \psi\right)_{L^{2}}= & \left(-(\rho u \cdot \nabla u+2 \zeta \operatorname{rot} w)_{t}, \psi\right)_{L^{2}}-\left((\mu+\zeta) \nabla u_{t}, \nabla \psi\right)_{L^{2}} \\
& -\left((\mu+\lambda-\zeta) \operatorname{div} u_{t}-p(\rho)_{t}, \operatorname{div} \psi\right)_{L^{2}} .
\end{aligned}
$$

Using the regularity (38) of $(\rho, u, w)$, we show the right-hand side of (42) is bounded above by $A_{2}(t)|\psi|_{H_{0}^{1}}$ for some positive function $A_{2}(t) \in L^{2}(0, T)$. Hence by the similar argument of $\rho w_{t}$, we see that $\left(\rho u_{t}\right)_{t} \in L^{2}\left(0, T ; H^{-1}\right)$, and then $\rho u_{t} \in C\left([0, T] ; L^{2}\right)$. Therefore, we conclude that for each $t \in[0, T], u=u(t) \in D_{0}^{1} \cap D^{2}$ is a solution of the elliptic system:

$$
-(\mu+\lambda) \Delta u-(\mu+\lambda-\zeta) \nabla \operatorname{div} u=G_{2}-\rho_{v} \cdot \nabla u,
$$

where $G_{2}=-\rho u_{t}-\nabla p(\rho)+2 \zeta \operatorname{rot} w \in C\left([0, T] ; L^{2}\right)$.

Now, we will show that $u \in C\left([0, T] ; D^{2}\right)$. In view of the elliptic regularity estimate (36), we have

$$
|u(t)-u(s)|_{D^{2}} \leq C|u(t)-u(s)|_{L^{2}}+C\left|G_{2}(t)-G_{2}(s)\right|_{L^{2}}+C|\rho v \cdot \nabla u(t)-\rho v \cdot \nabla u(s)|_{L^{2}} .
$$


To estimate the third term in the right-hand side of (43), using the estimate (38), similarly as (41), we get:

$$
\begin{aligned}
C|\rho v \cdot \nabla u(t)-\rho v \cdot \nabla u(s)|_{L^{2}} \leq & C\left(|\rho(t)-\rho(s)|_{L^{\infty}}+|\nabla v(t)-\nabla v(s)|_{L^{2}}+|\nabla u(t)-\nabla u(s)|_{L^{2}}\right) \\
& +\frac{1}{2}|u(t)-u(s)|_{D^{2}} .
\end{aligned}
$$

Substituting this into (43), we conclude the continuity of $u$ in $D^{2}$. This completes the proof of the continuity.

Finally, we prove the uniqueness of solutions satisfying the regularity (38). Let ( $\rho_{1}$, $\left.u_{1}, w_{1}\right)$ and $\left(\rho_{2}, u_{2}, w_{2}\right)$ be two strong solutions to the problem (8) and (2)-(4). Denote

$$
\bar{\rho}=\rho_{1}-\rho_{2}, \quad \bar{u}=u_{1}-u_{2}, \quad \bar{w}=w_{1}-w_{2} .
$$

Then, it follows from $(8)_{1}$ that

$$
\frac{\mathrm{d}}{\mathrm{d} t} \int|\bar{\rho}|^{2} \mathrm{~d} x \leq \int|\operatorname{div} v||\bar{\rho}|^{2} \mathrm{~d} x .
$$

Since $\nabla v \in L^{2}\left(0, T ; W^{1, q_{0}}\right)$ and $\bar{\rho}(0)=0$, we conclude from Gronwall's inequality that $|\bar{\rho}|_{L^{2}}=0$, i.e., $\rho_{1}=\rho_{2}$ in $(0, T) \times \Omega$. Next, we choose a cut-off function $\varphi \in C_{c}^{\infty}\left(\mathbb{R}^{3}\right)$ such that

$$
\varphi(x)=\left\{\begin{array}{l}
1, \text { if }|x| \leq 1 \\
0, \text { if }|x| \geq 2
\end{array}\right.
$$

Define $\phi_{R}(x)=\phi(x / R)$ for $x \in \mathbb{R}^{3}$. From $(8)_{2}$ and using the uniqueness of $\rho$, we deduce that

$$
\rho_{1} \bar{w}_{t}+\rho_{1} u \cdot \nabla \bar{w}+4 \zeta \bar{w}=\mu^{\prime} \Delta \bar{w}+\left(\mu^{\prime}+\lambda^{\prime}\right) \nabla \operatorname{div} \bar{w} .
$$

Then multiplying the above equation by $\varphi_{R}^{2} \bar{w}$, integrating over $(0, T) \times \Omega$, and letting $R \rightarrow \infty$, we easily get

$$
\frac{1}{2} \int \rho_{1}|\bar{w}|^{2}(t) \mathrm{d} x+\int_{0}^{t} \int\left(\mu^{\prime}|\nabla \bar{w}|^{2}+\left(\mu^{\prime}+\lambda^{\prime}\right)|\operatorname{div} \bar{w}|^{2}+4 \zeta|\bar{w}|^{2}\right) \mathrm{d} x \mathrm{~d} s=\frac{1}{2} \int \rho_{1}|\bar{w}|^{2}(0) \mathrm{d} x .
$$

Hence, we deduce that $\left|\sqrt{\rho_{1}} \bar{w}\right|_{L^{2}}=0,|\bar{w}|_{L^{2}}=0$ and $|\nabla \bar{w}|_{L^{2}}=0$ in $(0, T)$, due to $\bar{w}(0)=0$. Therefore, we conclude that $\bar{w}=0$ in $(0, T) \times \Omega$.

Similarly, from the uniqueness of $\rho$, and $(8)_{3}$, we get

$$
\rho_{1} \bar{u}_{t}+\rho_{1} v \nabla \bar{u}=(\mu+\zeta) \Delta \bar{u}+(\mu+\lambda-\zeta) \nabla \operatorname{div} \bar{u} .
$$

Then multiplying it by $\varphi_{R}^{2} \bar{u}$ integrating over $(0, T) \times \Omega$, and letting $R \rightarrow \infty$, we obtain

$$
\frac{1}{2} \int \rho_{1}|\bar{u}|^{2}(t) \mathrm{d} x+\int_{0}^{t} \int\left((\mu+\zeta)|\nabla \bar{u}|^{2}+(\mu+\lambda-\zeta)|\operatorname{div} \bar{u}|^{2}\right) \mathrm{d} x \mathrm{~d} s=\frac{1}{2} \int \rho_{1}|\bar{u}|^{2}(0) \mathrm{d} x .
$$

Due to $\bar{u}(0)=0$, we get $\left|\sqrt{\rho_{1}} \bar{u}\right|_{L^{2}}=0,|\nabla \bar{u}|_{L^{2}}=0$ in $(0, T)$. Then $\bar{u}=0$ in $(0, T) \times \Omega$.

This completes the proof of the Theorem 2 .

\section{A local existence result for positive densities}

In this section, we assume also that $\Omega$ is a bounded domain in $\mathbb{R}^{3}$ with smooth boundary and prove a local (in time) existence result on strong solutions with positive densities to the original nonlinear problem (1)-(4). 
Proposition 1 Assume that $p=a \rho^{\gamma}(a>0, \gamma>1)$, and the data $\left(\rho_{0}, u_{0}, w_{0}\right)$ satisfies the regularity conditions:

$$
\rho_{0} \in H^{1} \cap W^{1, q}, \quad\left(u_{0}, w_{0}\right) \in D_{0}^{1} \cap D^{2},
$$

for some $q$ with $3<q<\infty$ and compatibilities (6)-(7). Assume further that $\rho_{0} \geq \delta$ in $\Omega$ for some constant $\delta>0$. Then there exist a time $T_{*} \in(0, T)$ and a unique strong solution $(\rho, u, w)$ to the nonlinear problem (1)-(3) such that

$$
\begin{aligned}
& \rho \in C\left([0, T] ; H^{1} \cap W^{1, q_{0}}\right),(u, w) \in C\left(\left[0, T_{*}\right] ; D_{0}^{1} \cap D^{2}\right) \cap L^{2}\left(0, T_{*} ; D^{2, q_{0}}\right), \\
& \rho_{t} \in C\left(\left[0, T_{*}\right] ; L^{2} \cap L^{q_{0}}\right),\left(u_{t}, w_{t}\right) \in L^{2}\left(0, T_{*} ; D_{0}^{1}\right), \\
& \left(\sqrt{\rho} u_{t}, \sqrt{\rho} w_{t}\right) \in L^{\infty}\left(0, T_{*} ; L^{2}\right),
\end{aligned}
$$

where $q 0=\min (6, q)$. Furthermore, we have the following estimates:

$$
\begin{aligned}
& \underset{\substack{0<t<T_{*} \\
\text { ess sup }}}{T_{*}}\left(|\rho(t)|_{H^{1} \cap W^{1, q_{0}}}+\left|\rho_{t}(t)\right|_{L^{2} \cap L^{q_{0}}}+|(u(t), w(t))|_{D_{0}^{1} \cap D^{2}}+\left|\left(\sqrt{\rho} u_{t}, \sqrt{\rho} w_{t}\right)\right|_{L^{2}}\right) \\
& +\int_{0}^{T^{2}}\left(|(u(t), w(t))|_{D^{2, q_{0}}}^{2}+\left|\left(u_{t}, w_{t}\right)\right|_{D_{0}^{1}}^{2}\right) \mathrm{d} t \leq C .
\end{aligned}
$$

The constant $C$ and the local time $T_{*}$ in (44) are independent of $\delta$.

To prove the proposition, we first construct approximate solutions, inductively, as follows:

- first define $u^{0}=0$, and

- assume that $u^{k-1}$ was defined for $k \geq 1$, let $\left(\rho^{k}, u^{k}, w^{k}\right)$ be the unique solution to the following initial boundary value problem:

$$
\begin{aligned}
& \rho_{t}^{k}+u^{k-1} \cdot \nabla \rho^{k}+\rho^{k} \operatorname{div} u^{k-1}=0, \\
& \rho^{k} w_{t}^{k}+\rho^{k} u^{k-1} \cdot \nabla w^{k}+4 \zeta w^{k}=\mu^{\prime} \Delta w^{k}+\left(\mu^{\prime}+\lambda^{\prime}\right) \nabla \operatorname{div} w^{k}+2 \zeta \operatorname{rot} u^{k-1}, \\
& \rho^{k} u_{t}^{k}+\rho^{k} u^{k-1} \cdot \nabla u^{k}+\nabla p^{k}=(\mu+\zeta) \Delta u^{k}+(\mu+\lambda-\zeta) \nabla \operatorname{div} u^{k}+2 \zeta \operatorname{rot} w^{k}, \\
& \left.\rho\right|_{t=0}=\rho_{0},\left.\quad u^{k}\right|_{t=0}=u_{0},\left.\quad w^{k}\right|_{t=0}=w_{0},\left.\quad u^{k}\right|_{\partial \Omega}=\left.w^{k}\right|_{\partial \Omega}=0, \\
& \rho^{k}(t, x) \rightarrow 0, u^{k}(t, x) \rightarrow 0, w^{k}(t, x) \rightarrow 0 \text { as }|x| \rightarrow \infty,(t, x) \in(0, T) \times \Omega .
\end{aligned}
$$

The existence of a global strong solution $\left(\rho^{k}, u^{k}, w^{k}\right)$ with the regularity (12) to the linearized problem (45)-(49) was proved in the previous section.

From now on, we derive uniform bounds on the approximate solutions and then prove the convergence of the approximate solutions to a strong solutions of the original nonlinear problem.

\subsection{Uniform bounds}

Let $K \geq 1$ be a fixed large integer, and let us introduce an auxiliary function $\Phi_{K}(t)$, defined by:

$$
\Phi_{K}(t)=\max _{1 \leq k \leq K} \sup _{0 \leq s \leq t}\left(1+\left|\rho^{k}(s)\right|_{H^{1} \cap W^{1, q_{0}}}^{2}+\left|\left(\sqrt{\rho^{k}} u_{t^{\prime}}^{k} \sqrt{\rho^{k}} w_{t}^{k}\right)\right|_{L^{2}}^{2}+\left|w^{k}(s)\right|_{L^{2}}^{2}+\left|\left(u^{k}(s), w^{k}(s)\right)\right|_{D_{0}^{1}}^{2}\right) .
$$


Then we estimate each term of $\Phi_{K}$ in terms of some integrals of $\Phi_{K}$, apply arguments of Gronwall-type and thus prove that $\Phi_{K}$ is locally bounded. First, notice that

$$
\left|\rho^{k}(t)\right|_{L^{q}}+\left|p^{k}(t)\right|_{L^{q}} \leq C \Phi_{K}(t), \quad \text { for all } 1 \leq q \leq \infty .
$$

Then, we estimate $\left|\left(u^{k}, w^{k}\right)\right|_{H^{2}}$ by $\Phi_{K^{\prime}}$.

- Estimate $\left|w^{k}\right|_{H^{2}}$

Observe that for any $t \in[0, T], w^{k}=w^{k}(t) \in D_{0}^{1} \cap D^{2}$ is a solution of the elliptic system:

$$
-\mu^{\prime} \Delta w^{k}-\left(\mu^{\prime}+\lambda\right) \nabla \operatorname{div} w^{k}=-\rho^{k} w_{t}^{k}-\rho^{k} \mu^{k-1} \cdot \nabla w^{k}-4 \zeta w^{k}+2 \zeta \operatorname{rot} u^{k-1} .
$$

Hence we deduce from the elliptic regularity result that

$$
\begin{aligned}
\left|\nabla^{2} w^{2}\right|_{L^{2}} & \leq C\left(\left|\rho^{k} w_{t}^{k}\right|_{L^{2}}+\left|\rho^{k} u^{k-1} \cdot \nabla w^{k}\right|_{L^{2}}+\left|w^{k}\right|_{L^{2}}+\left|\nabla u^{k-1}\right|_{L^{2}}+\left|\nabla w^{k}\right|_{L^{2}}\right) \\
& \leq C\left(\left|\rho^{k}\right|_{L^{\infty}}\left|\sqrt{\rho^{k}} w_{t}^{k}\right|_{L^{2}}+\left|\rho^{k}\right|_{L^{\infty}}\left|\nabla u^{k-1}\right|_{L^{2}}\left|\nabla w^{k}\right|_{L^{2}}^{1 / 2}\left|\nabla w^{k}\right|_{H^{1}}^{1 / 2}+\left|\nabla u^{k-1}\right|_{L^{2}}+\left|\nabla w^{k}\right|_{L^{2}}\right) \\
& \leq C \Phi_{K}^{5 / 2}+\frac{1}{2}\left|\nabla w^{k}\right|_{H^{1}} .
\end{aligned}
$$

Thus we conclude

$$
\left|\nabla w^{k}\right|_{H^{1}} \leq C \Phi_{K}^{5 / 2}
$$

- Estimate $\left|u^{k}\right|_{H^{2}}$

Similar as the estimate of $\left|w^{k}\right|_{H^{2}}$ We see that for any $t \in[0, T], u^{k}=u^{k}(t) \in D_{0}^{1} \cap D^{2}$ is a solution of the elliptic system:

$$
(\mu+\zeta) \Delta u^{k}+(\mu+\lambda-\zeta) \nabla \operatorname{div} u^{k}=-\rho u_{t}^{k}-\rho u^{k-1} \cdot \nabla u^{k}-\nabla p^{k}+2 \zeta \operatorname{rot} w^{k} .
$$

Hence, we have

$$
\begin{aligned}
& \left|\nabla^{2} u^{k}\right|_{L^{2}} \\
\leq & C\left(\left|\rho^{k} u_{t}^{k}\right|_{L^{2}}+\left|\rho^{k} u^{k-1} \cdot \nabla u^{k}\right|_{L^{2}}+\left|\nabla p^{k}\right|_{L^{2}}+\left|\nabla w^{k}\right|_{L^{2}}+\left|\nabla u^{k}\right|_{L^{2}}\right) \\
\leq & C\left(\left|\rho^{k}\right|_{L^{\infty}}^{1 / 2}\left|\sqrt{\rho^{k}} u_{t}^{k}\right|_{L^{2}}+\left|\rho^{k}\right|_{L^{\infty}}\left|u^{k-1}\right|_{L^{6}}\left|\nabla u^{k}\right|_{L^{3}}+\left|\rho^{k}\right|_{L^{\infty}}^{\gamma-1}\left|\nabla \rho^{k}\right|_{L^{2}}+\left|\nabla w^{k}\right|_{L^{2}}+\left|\nabla u^{k}\right|_{L^{2}}\right) \\
\leq & C\left(\left|\rho^{k}\right|_{L^{\infty}}^{1 / 2}\left|\sqrt{\rho^{k} u_{t}^{k}}\right|_{L^{2}}+\left|\rho^{k}\right|_{L^{\infty}}\left|\nabla u^{k-1}\right|_{L^{2}}\left|\nabla u^{k}\right|_{L^{2}}^{1 / 2}\left|\nabla u^{k}\right|_{H^{1}}^{1 / 2}+\left|\rho^{k}\right|_{L^{\infty}}^{\gamma-1}\left|\nabla \rho^{k}\right|_{L^{2}}+\left|\nabla w^{2}\right|_{L^{2}}+\left|\nabla u^{k}\right|_{L^{2}}\right) \\
\leq & C \Phi_{K}^{\gamma+5 / 2}+\frac{1}{2}\left|\nabla u^{k}\right|_{H^{1}} .
\end{aligned}
$$

Thus we conclude

$$
\left|\nabla u^{k}\right|_{H^{1}} \leq C \Phi_{K}^{\gamma+5 / 2}
$$

- Estimate $\left|w^{k}\right|_{D_{0}^{1}}$

Multiplying (46) by $w_{t}^{k}$, and integrating over $\Omega$, using Young's inequality we obtain:

$$
\begin{aligned}
& \frac{1}{2} \int \rho^{k}\left|w_{t}^{k}\right|^{2} \mathrm{~d} x+\frac{\mathrm{d}}{\mathrm{d} t} \int\left(\frac{\mu^{\prime}}{2}\left|\nabla w^{k}\right|^{2}+\frac{\mu^{\prime}+\lambda^{\prime}}{2}\left|\operatorname{div} w^{k}\right|+2 \zeta\left|w^{k}\right|^{2}\right) \mathrm{d} x \\
\leq & \frac{\mathrm{d}}{\mathrm{d} t} \int 2 \zeta \operatorname{rot} u^{k-1} w^{k} \mathrm{~d} x+\int\left(\rho\left|u^{k-1}\right|^{2}\left|\nabla w^{k}\right|^{2}+2 \zeta\left|\nabla u_{t}^{k-1}\right|\left|w^{k}\right| \mathrm{d} x .\right.
\end{aligned}
$$


Integrating the above inequality we get

$$
\begin{aligned}
& \int_{0}^{t}\left|\sqrt{\rho^{k}} w_{t}^{k}\right|_{L^{2}}^{2} \mathrm{~d} s+\left|\nabla w^{k}\right|_{L^{2}}^{2}+\left|w^{k}\right|_{L^{2}}^{2} \\
& \leq C\left|\nabla u^{k-1}\right|_{L^{2}}^{2}+\int_{0}^{t}\left(\left|\rho^{k}\right|_{L^{\infty}}\left|u^{k-1}\right|_{L^{6}}^{2}\left|\nabla w^{k}\right|_{L^{3}}^{2}+\eta\left|\nabla u_{t}^{k-1}\right|_{L^{2}}^{2}+C\left|w^{k}\right|_{L^{2}}^{2}\right) \mathrm{d} s .
\end{aligned}
$$

To estimate the right-hand side of (52), we first observe that $\left|\rho^{k}\right|_{L^{\infty}} \leq C \Phi_{K}^{1 / 2},\left|u^{k-1}\right|_{L^{6}}^{2} \leq C\left|\nabla u^{k-1}\right|_{L^{2}}^{2} \leq C \Phi_{K}$, together with the estimate $\left|u^{k}\right|_{H^{2}}$ we conclude that:

$$
\int_{0}^{t}\left|\sqrt{\rho^{k}} w_{t}^{2}\right|_{L^{2}}^{2} \mathrm{~d} s+\left|\nabla w^{k}\right|_{L^{2}}^{2}+\left|w^{k}\right|_{L^{2}}^{2} \leq C \int_{0}^{t} \Phi_{K}^{4} \mathrm{~d} s+\eta\left|\nabla u_{t}^{k-1}\right|_{L^{2}}^{2} .
$$

- Estimate $\left|\sqrt{\rho^{k}} w_{t}^{k}\right|_{L^{2}}$

Differentiating (46) with respect to $t$, we obtain

$$
\begin{aligned}
& \rho^{k} w_{t t}^{k}+\rho^{k} u^{k-1} \cdot \nabla w_{t}^{k}-4 \zeta w_{t}^{k}-\mu^{\prime} \Delta w_{t}^{k}-\left(\mu^{\prime}+\lambda^{\prime}\right) \nabla \operatorname{div} w^{k} \\
= & 2 \zeta \operatorname{rot} u_{t}^{k-1}-\rho_{t}^{k} w_{t}^{k}-\rho_{t}^{k} u^{k-1} \cdot \nabla w^{k}-\rho^{k} u^{k-1} \cdot \nabla w^{k} .
\end{aligned}
$$

Multiplying this by $w_{t}^{k}$ and integrating over $\Omega$, we obtain:

$$
\begin{aligned}
& \frac{\mathrm{d}}{\mathrm{d} t} \int \rho^{k}\left|w_{t}^{k}\right|^{2} \mathrm{~d} x+\int\left(4 \zeta\left|w_{t}^{k}\right|^{2}+\mu^{\prime}\left|\nabla w_{t}^{k}\right|^{2}+\left(\mu^{\prime}+\lambda^{\prime}\right)\left|\operatorname{div} w_{t}^{k}\right|^{2}\right) \mathrm{d} x \\
\leq & 2 \zeta \int\left(\left|\nabla u_{t}^{k-1}\right|\left|w_{t}^{k}\right| \mathrm{d} x+\int 2 \rho^{k}\left|u^{k-1}\right|\left|w_{t}^{k}\right|\left|\nabla w_{t}^{k}\right|+p^{k}\left|u_{t}^{k-1}\right|\left|\nabla w^{k}\right|\left|w_{t}^{k}\right|\right. \\
& +\rho^{k}\left|u^{k-1}\right|\left|\nabla u^{k-1}\right|\left|\nabla w^{k}\right|\left|w_{t}^{k}\right|+\rho^{k}\left|u^{k-1}\right|^{2}\left|\nabla^{2} w^{k}\right|\left|w_{t}^{k}\right| \\
& +\rho^{k}\left|u^{k-1}\right|^{2}\left|\nabla w^{k}\right|\left|\nabla w_{t}^{k}\right| \mathrm{d} x \sum_{i=1}^{6} I I I_{i} .
\end{aligned}
$$

Using Sobolev inequality and Young's inequality (with $\varepsilon$ ) repeatedly, we have:

$$
\begin{aligned}
I I I_{1} & =2 \zeta \int\left|\nabla u_{t}^{k-1}\right|\left|w_{t}^{k}\right| \mathrm{d} x \leq \frac{\zeta}{2}\left|\nabla u_{t}^{k-1}\right|_{L^{2}}^{2}+2 \zeta\left|w_{t}^{k}\right|_{L^{2}}^{2} ; \\
I I I_{2} & =2 \int \rho^{k}\left|u^{k-1}\right|\left|w_{t}^{k}\right|\left|\nabla w_{t}^{k}\right| \mathrm{d} x \leq 2\left|\rho^{k}\right|_{L^{\infty}}^{1 / 2}\left|u^{k-1}\right|_{L^{6}}\left|\sqrt{\rho^{k}} w_{t}^{k}\right|_{L^{3}}\left|\nabla w_{t}^{k}\right|_{L^{2}} \\
& \leq 2\left|\rho^{k}\right|_{L^{\infty}}^{1 / 2}\left|\nabla u^{k-1}\right|_{L^{2}}\left|\sqrt{\rho^{k}} w_{t}^{k}\right|_{L^{2}}^{1 / 2}\left|\sqrt{\rho^{k}} w_{t}^{k}\right|_{L^{6}}^{1 / 2}\left|\nabla w_{t}^{k}\right|_{L^{2}} \\
& \leq 2\left|\rho^{k}\right|_{L^{\infty}}^{3 / 4}\left|\nabla u^{k-1}\right|_{L^{2}}\left|\sqrt{\rho^{k}} w_{t}^{k}\right|_{L^{2}}^{1 / 2}\left|\nabla w_{t}^{k}\right|_{L^{2}}^{3 / 2} \leq C \Phi_{K}^{9 / 2}+\varepsilon\left|\nabla w_{t}^{k}\right|_{L^{2}}^{2} ; \\
I I I_{3} & =\left.\left.\left.\int \rho^{k} u_{t}^{k-1}|| \nabla w^{k}|| w_{t}^{k}|\mathrm{~d} x \leq| \rho^{k}\right|_{L^{\infty}} ^{1 / 2}\right|_{t} ^{k-1}\right|_{L^{6}}\left|\sqrt{\rho^{k}} w_{t}^{k}\right|_{L^{3}}\left|\nabla w^{k}\right|_{L^{2}} \\
& \leq\left|\rho^{k}\right|_{L^{\infty}}^{1 / 2}\left|\nabla u_{t}^{k-1}\right|_{L^{2}}\left|\sqrt{\rho^{k}} w_{t}^{k}\right|_{L^{2}}^{1 / 2}\left|\sqrt{\rho^{k}} w_{t}^{k}\right|_{L^{6}}^{1 / 2}\left|\nabla w^{k}\right|_{L^{2}} \\
& \leq\left.\left.\left|\rho^{k}\right|_{L^{\infty}}^{3 / 4}\left|\nabla u_{t}^{k-1}\right|_{L^{2}}\left|\sqrt{\rho^{k}} w_{t}^{k}\right|_{L^{2}}^{1 / 2}\left|\nabla w_{t}^{k}\right|_{L^{2}}^{1 / 2}\right|_{L^{k}}\right|_{L^{2}} \\
& \leq C\left|\rho^{k}\right|_{L^{\infty}}^{3}\left|\sqrt{\rho^{k}} w_{t}^{k}\right|_{L^{2}}^{2}\left|\nabla w^{k}\right|_{L^{2}}^{4}+\varepsilon\left|\nabla w_{t}^{k}\right|_{L^{2}}^{2}+\eta\left|\nabla u_{t}^{k-1}\right|_{L^{2}}^{2} \\
& \leq C \Phi_{K}^{9 / 2}+\varepsilon\left|\nabla w_{t}^{k}\right|_{L^{2}}^{2}+\eta\left|\nabla u_{t}^{k-1}\right|_{L^{2}}^{2}
\end{aligned}
$$




$$
\begin{aligned}
I I_{4} & =\int \rho^{k}\left|u^{k-1}\right|\left|\nabla u^{k-1}\right|\left|\nabla w^{k}\right|\left|w_{t}^{k}\right| \mathrm{d} x \\
& \leq\left|\rho^{k}\right|_{L^{\infty}}\left|u^{k-1}\right|_{L^{6}}\left|\nabla u^{k-1}\right|_{L^{3}}\left|\nabla w^{k}\right|_{L^{3}}\left|w_{t}^{k}\right|_{L^{6}} \\
& \leq C\left|\rho^{k}\right|_{L^{\infty}}\left|\nabla u^{k-1}\right|_{L^{2}}^{3 / 2}\left|\nabla u^{k-1}\right|_{H^{1}}^{1 / 2}\left|\nabla w^{k}\right|_{L^{2}}^{1 / 2}\left|\nabla w^{k}\right|_{H^{1}}^{1 / 2}\left|\nabla w_{t}^{k}\right|_{L^{2}} \\
& \leq C\left|\rho^{k}\right|_{L^{\infty}}^{2}\left|\nabla u^{k-1}\right|_{L^{2}}^{3}\left|\nabla u^{k-1}\right|_{H^{1}}\left|\nabla w^{k}\right|_{L^{2}}\left|\nabla w^{k}\right|_{H^{1}}+\varepsilon\left|\nabla w_{t}^{k}\right|_{L^{2}}^{2} \\
& \leq C \Phi_{K}^{\gamma+8}+\varepsilon\left|\nabla w_{t}^{k}\right|_{L^{2}}^{2} ; \\
I I I_{5} & =\left.\left.\int \rho^{k}\right|^{k-1}\right|^{2}\left|\nabla^{2} w^{k}\right|\left|w_{t}^{k}\right| \mathrm{d} x \\
& \leq\left|\rho^{k}\right|_{L^{\infty}}\left|u^{k-1}\right|_{L^{6}}^{2}\left|\nabla^{2} w^{k}\right|_{L^{2}}\left|w_{t}^{k}\right|_{L^{6}} \\
& \leq C\left|\rho^{k}\right|_{L^{\infty}}^{2}\left|\nabla u^{k-1}\right|_{L^{2}}^{4}\left|\nabla^{2} w^{k}\right|_{L^{2}}^{2}+\varepsilon\left|\nabla w_{t}^{k}\right|_{L^{2}}^{2} \\
& \leq C \Phi_{K}^{8}+\varepsilon\left|\nabla w_{t}^{k}\right|_{L^{2}}^{2} ; \\
I I I_{6} & =\int \rho^{k}\left|u^{k-1}\right|^{2}\left|\nabla w^{k}\right|\left|\nabla w_{t}^{k}\right| \mathrm{d} x \leq\left|\rho^{k}\right|_{L^{\infty}}\left|\nabla u^{k-1}\right|_{L^{2}}^{2}\left|\nabla w^{k}\right|_{H^{1}}\left|\nabla w_{t}^{k}\right|_{L^{2}}^{2} \\
& \leq C \Phi_{K}^{8}+\varepsilon\left|\nabla w_{t}^{k}\right|_{L^{2}}^{2} \cdot
\end{aligned}
$$

Substituting all these estimates into (54) and choosing $\varepsilon, \eta>0$ small enough, we obtain that:

$$
\frac{\mathrm{d}}{\mathrm{d} t} \int \rho^{k}\left|w_{t}^{k}\right|^{2} \mathrm{~d} x+\zeta\left|w_{t}^{k}\right|_{L^{2}}^{2}+\left|\nabla w_{t}^{k}\right|_{L^{2}}^{2} \leq C \Phi_{K}^{\gamma+8}+\left(\frac{\zeta}{2}+\eta\right)\left|\nabla u_{t}^{k-1}\right|_{L^{2}}^{2} .
$$

Integrating over $(\tau, t)$ for fixed $\tau>0$, we have

$$
\begin{aligned}
& \int \rho^{k}\left|w_{t}^{k}\right|^{2} \mathrm{~d} x+\int_{\tau}^{t}\left(2 \zeta\left|w_{t}^{k}\right|_{L^{2}}^{2}+\frac{\mu^{\prime}}{2}\left|\nabla w_{t}^{k}\right|_{L^{2}}^{2}\right) \mathrm{d} s \\
\leq & C \int_{\tau}^{t}\left(\Phi^{\gamma+8}\left(\frac{\zeta}{2}+\eta\right)\left|\nabla u_{t}^{k-1}\right|_{L^{2}}^{2}\right) \mathrm{d} s+\int \rho^{k}\left|w_{t}^{k}\right|^{2}(\tau) \mathrm{d} x .
\end{aligned}
$$

To estimate $\int \rho^{k}\left|w_{t}^{k}\right|^{2}(\tau) \mathrm{d} x$ as $\tau \rightarrow 0$, we multiplying (46) by $w_{t}^{k}$, integrate over $\Omega$, we have:

$$
\int \rho^{k}\left|w_{t}^{k}\right|^{2} \mathrm{~d} x=-\int \rho^{k} u^{k-1} \cdot \nabla w^{k} w_{t} \mathrm{~d} x+\int\left(\mu^{\prime} \Delta w^{k}+\left(\mu^{\prime}+\lambda^{\prime}\right) \nabla \operatorname{div} w^{k}+2 \zeta \operatorname{rot} u^{k-1}-4 \zeta w^{k}\right) w_{t}^{k} \mathrm{~d} x
$$

Using Young's inequality, together with the compatibility condition (7), we have

$$
\begin{aligned}
& \lim _{\tau \rightarrow 0} \frac{1}{2} \int \rho^{k}\left|w_{t}^{k}\right|^{2} \mathrm{~d} x \leq C\left(\int \rho^{k}\left|u^{k-1}\right|^{2}\left|\nabla w^{k}\right|^{2} \mathrm{~d} x\right. \\
& \left.+\int \rho^{-1}\left(\mu^{\prime} \Delta w^{k}+\left(\mu^{\prime}+\lambda^{\prime}\right) \nabla \operatorname{div}+2 \zeta \operatorname{rot} u^{k-1}-4 \zeta w^{k}\right)^{2} \mathrm{~d} x\right) \leq C .
\end{aligned}
$$

Substituting the above estimate into (56), we conclude that

$$
\int \rho^{k}\left|w_{t}^{k}\right|^{2} \mathrm{~d} x=-\int \rho^{k} u^{k-1} \cdot \nabla w^{k} w_{t} \mathrm{~d} x \leq C\left(1+\int_{0}^{t} \Phi^{\gamma+8} \mathrm{~d} s\right)+\left(\frac{\zeta}{2}+\eta\right) \int_{0}^{t}\left|\nabla u_{t}^{k-1}\right|_{L^{2}}^{2} \mathrm{~d} s .
$$


- Estimate $\left|u^{k}\right|_{D_{0}^{1}}$

Multiplying (47) by $u_{t}^{k}$ and integrating over $\Omega$, we obtain:

$$
\begin{aligned}
& \frac{1}{2} \int \rho^{k}\left|u_{t}^{k}\right|^{2} \mathrm{~d} x+\frac{\mathrm{d}}{\mathrm{d} t} \int\left(\frac{\mu+\zeta}{2}\left|\nabla u^{k}\right|^{2}+\frac{\mu+\lambda-\zeta}{2}\left|\operatorname{div} u^{k}\right|^{2}\right) \mathrm{d} x \\
\leq & \frac{\mathrm{d}}{\mathrm{d} t} \int\left(p^{k} \mathrm{div} u^{k}+2 \zeta \operatorname{rot} u^{k} w^{k}\right) \mathrm{d} x+\int \rho^{k}\left|u^{k-1}\right|^{2}\left|\nabla u^{k}\right|^{2} \mathrm{~d} x+\int\left|p_{t}^{k}\right|\left|\nabla u^{k}\right| \mathrm{d} x+2 \zeta \int\left|w_{t}^{k}\right|\left|\nabla u^{k}\right| \mathrm{d} x .
\end{aligned}
$$

Using (45), we have

$$
p_{t}^{k}+u^{k-1} \cdot \nabla p^{k}+\gamma p^{k} \operatorname{div} u^{k-1}=0 .
$$

Integrating (58) over $(0, t)$, using (59) and Young's inequality, we have

$$
\begin{aligned}
& \frac{1}{2} \int_{0}^{t} \int \rho^{k}\left|u_{t}^{k}\right|^{2} \mathrm{~d} x \mathrm{~d} s+\frac{\mu+\zeta}{2}\left|\nabla u^{k}\right|_{L^{2}}^{2} \\
\leq & C+C \int\left|w^{k}\right|^{2} \mathrm{~d} x+\frac{\zeta}{2} \int\left|\nabla u^{k}\right|^{2} \mathrm{~d} x+C \int\left(p^{k}\right)^{2} \mathrm{~d} x \\
& +\int_{0}^{t} \int \rho^{k}\left|u^{k-1}\right|^{2}\left|\nabla u^{k}\right|^{2} \mathrm{~d} x \mathrm{~d} s+\int_{0}^{t} \int\left|u^{k}\right|\left|\nabla p^{k}\right|\left|\nabla u^{k}\right| \mathrm{d} x \mathrm{~d} s \\
& +\int_{0}^{t} \int \gamma\left|p^{k}\right|\left|\nabla u^{k-1}\right|\left|\nabla u^{k}\right| \mathrm{d} x \mathrm{~d} s+2 \zeta \int_{0}^{t} \int\left|w_{t}^{k}\right|\left|\nabla u^{k}\right| \mathrm{d} x \mathrm{~d} s \\
= & C+C \Phi_{K}+\sum_{i=1}^{5} I V_{i} .
\end{aligned}
$$

To estimate the right-hand side of (60), with the help of Sobolev inequality and Young's inequality (with $\varepsilon$ ), we have:

$$
\begin{aligned}
I V_{1} & =\int\left(p^{k}\right)^{2} \mathrm{~d} x=\int p^{k}(0)^{2} \mathrm{~d} x+\int_{0}^{t} \frac{\partial}{\partial s}\left(\int p^{k}(s)^{2} \mathrm{~d} x\right) \mathrm{d} s \\
& \leq C+2 \int_{0}^{t} \int\left(a^{2} \gamma\left(\rho^{k}\right)^{2 \gamma-1}\left|\nabla \rho^{k}\right|\left|u^{k-1}\right|+a^{2} \gamma\left(\rho^{k}\right)^{2}\left|\nabla u^{k-1}\right|\right) \mathrm{d} x \mathrm{~d} s \\
& \leq C+C \int_{0}^{t}\left|\rho^{k}\right|_{L^{\infty}}^{2(\gamma-1)}\left|\rho^{k}\right|_{L^{3}}\left|\nabla \rho^{k}\right|_{L^{2}}\left|u^{k-1}\right|_{L^{6}}+\left|\rho^{k}\right|_{L^{\infty}}^{2 \gamma-1}\left|\rho^{k}\right|_{L^{2}}\left|\nabla u^{k-1}\right|_{L^{2}} \mathrm{~d} s \\
& \leq C+C \int_{0}^{t}\left|\rho^{k}\right|_{L^{\infty}}^{2(\gamma-1)}\left|\rho^{k}\right|_{L^{2}}^{\alpha}\left|\rho^{k}\right|_{L^{q 0}}^{1-\alpha}\left|\nabla \rho^{k}\right|_{L^{2}}\left|\nabla u^{k-1}\right|_{L^{2}} \\
& +\left|\rho^{k}\right|_{L^{\infty}}^{2 \gamma-1}\left|\rho^{k}\right|_{L^{2}}\left|\nabla u^{k-1}\right|_{L^{2}} \mathrm{~d} \leq \leq+C \int_{0}^{t} \Phi_{K}^{2 \gamma+1 / 2} \mathrm{~d} s . \\
I V_{2} & =\left.\left.\int_{0}^{t} \int_{0}^{t} \rho^{k}\right|^{k-1}\right|^{2}\left|\nabla u^{k}\right|^{2} \mathrm{~d} x \mathrm{~d} s \leq \int_{0}^{t}\left|\rho^{k}\right|_{L^{\infty}}\left|u^{k-1}\right|_{L^{6}}^{2}\left|\nabla u^{k}\right|_{L^{3}}^{2} \\
& \leq C \int_{0}^{t}\left|\rho^{k}\right|_{L^{\infty}}\left|\nabla u^{k-1}\right|_{L^{2}}^{2}\left|\nabla u^{k}\right|_{L^{2}}\left|\nabla u^{k}\right|_{H^{1}} \mathrm{~d} s \leq C \int_{0}^{t} \Phi_{K}^{9 / 2} \mathrm{~d} s .
\end{aligned}
$$




$$
\begin{aligned}
I V_{3} & =\int_{0}^{t} \int\left|u^{k}\right|\left|\nabla p^{k}\right|\left|\nabla u^{k}\right| \mathrm{d} x \mathrm{~d} s \leq \int_{0}^{t}\left|u^{k}\right|_{L^{6}}\left|\nabla p^{k}\right|_{L^{3}}\left|\nabla u^{k}\right|_{L^{2}} \mathrm{~d} s \\
& \leq C \int_{0}^{t}\left|\nabla u^{k}\right|_{L^{2}}^{2}\left|\rho^{k}\right|_{L^{\infty}}^{\gamma-1}\left|\rho^{k}\right|_{L^{2}}^{\alpha}\left|\rho^{k}\right|_{L^{q 0}}^{1-\alpha} \mathrm{d} s \leq C \int_{0}^{t} \Phi_{K}^{\gamma+1 / 2} \mathrm{~d} s ; \\
I V_{4} & =\int_{0}^{t} \int_{0} \gamma\left|\rho^{k}\right| \nabla u^{k-1}\left|\nabla u^{k}\right| \mathrm{d} x \mathrm{~d} s \\
& \leq C \int_{0}^{t}\left|\rho^{k}\right|_{L^{\infty}}^{\gamma}\left|\nabla u^{k-1}\right|_{L^{2}}\left|\nabla u^{k}\right|_{L^{2}} \mathrm{~d} s \leq C \int_{0}^{t} \Phi_{K}^{\gamma+1} \mathrm{~d} s ; \\
I V_{5} & =2 \zeta \int_{0}^{t} \int\left|w_{t}^{k}\right|\left|\nabla u^{k}\right| \mathrm{d} x \mathrm{~d} s \leq \varepsilon \int_{0}^{t}\left|w_{t}^{k}\right|_{L^{2}}^{2} \mathrm{~d} s+C \int_{0}^{t}\left|\nabla u^{k}\right|_{L^{2}}^{2} \mathrm{~d} s \\
& \leq \varepsilon \int_{0}^{t}\left|w_{t}^{k}\right|_{L^{2}}^{2} \mathrm{~d} s+C \int_{0}^{t} \Phi_{K} \mathrm{~d} s .
\end{aligned}
$$

Substitute all the above estimates into (60), we obtain:

$$
\int_{0}^{t}\left|\sqrt{\rho^{k}} u_{t}^{k}\right|_{L^{2}}^{2} \mathrm{~d} s+\int_{0}^{t}\left|\nabla u^{k}\right|_{L^{2}}^{2} \mathrm{~d} s \leq C+C \int_{0}^{t} \Phi_{k}^{\max \{2 \gamma+1 / 2,9 / 2\}} \mathrm{d} s+\varepsilon \int_{0}^{t}\left|w_{t}^{k}\right|_{L^{2}}^{2} \mathrm{~d} s .
$$

- Estimate $\left|\sqrt{\rho^{K}} u_{t}^{k}\right|_{L^{2}}$

Differentiating (47) with respect to $t$, we obtain:

$$
\begin{aligned}
& \rho^{k} u_{t t}^{k}+\rho^{k} u^{k-1} \cdot \nabla u_{t}^{k}+\nabla p_{t}^{k}-(\mu+\zeta) \Delta u_{t}^{k}-(\mu+\lambda-\zeta) \nabla \operatorname{div} u_{t}^{k} \\
= & -\rho_{t}^{k} u_{t}^{k}-\rho_{t}^{k} u^{k-1} \cdot \nabla u^{k}-\rho^{k} u_{t}^{k-1} \cdot \nabla u^{k}+2 \zeta \operatorname{rot} w_{t}^{k} .
\end{aligned}
$$

Multiplying the equation by $u_{t}^{k}$, and integrating over $\Omega$, we get

$$
\begin{aligned}
& \quad \frac{1}{2} \frac{\mathrm{d}}{\mathrm{dt}} \int \rho^{k}\left|u_{t}^{k}\right| \mathrm{d} x+(\mu+\zeta) \int\left|\nabla u_{t}^{k}\right|^{2} \mathrm{~d} x+(\mu+\lambda-\zeta) \int\left|\operatorname{div} u_{t}^{k}\right|^{2} \mathrm{~d} x \\
& \leq 2 \int \rho^{k}\left|u^{k-1}\right|\left|\nabla u_{t}^{k}\right|\left|u_{t}^{k}\right| \mathrm{d} x+\int \rho^{k}\left|u^{k-1}\right|\left|\nabla u^{k-1}\right|\left|\nabla u^{k}\right|\left|u_{t}^{k}\right| \mathrm{d} x \\
& \quad+\int \rho^{k}\left|u^{k-1}\right|^{2}\left|\nabla^{2} u^{k}\right|\left|u_{t}^{k}\right| \mathrm{d} x+\int \rho^{k}\left|u^{k-1}\right|^{2}\left|\nabla u^{k}\right|\left|u_{t}^{k}\right| \mathrm{d} x \\
& \quad+\int \rho^{k}\left|u_{t}^{k-1}\right|\left|\nabla u^{k}\right|\left|u_{t}^{k}\right| \mathrm{d} x+2 \zeta \int\left|w_{t}^{k}\right|\left|\nabla u_{t}^{k}\right| \mathrm{d} x \\
& \quad+\int\left|\nabla p^{k}\right|\left|u^{k-1}\right|\left|\operatorname{div} u_{t}^{k}\right| \mathrm{d} x+\int p^{\prime}\left(\rho^{k}\right) \rho^{k}\left|\operatorname{div} u_{t}^{k-1}\right|\left|\operatorname{div} u_{t}^{k}\right| \mathrm{d} x=\sum_{i=1}^{8} V_{i} .
\end{aligned}
$$

Using Sobolev inequality and Young's inequality we have

$$
\begin{aligned}
V_{1} & =2 \int \rho^{k} u^{k-1}\left|\nabla u_{t}^{k}\right|\left|u_{t}^{k}\right| \mathrm{d} x \leq 2\left|\rho^{k}\right|_{L^{\infty}}^{1 / 2}\left|u^{k-1}\right|_{L^{6}}\left|\nabla u_{t}^{k}\right|_{L^{2}}\left|\sqrt{\rho^{k}} u_{t}^{k}\right|_{L^{3}} \\
& \leq 2\left|\rho^{k}\right|_{L^{\infty}}^{1 / 2}\left|\nabla u^{k-1}\right|_{L^{2}}\left|\nabla u_{t}^{k}\right|_{L^{2}}\left|\sqrt{\rho^{k}} u_{t}^{k}\right|_{L^{2}}^{1 / 2}\left|\sqrt{\rho^{k}} u_{t}^{k}\right|_{L^{6}}^{1 / 2} \\
& \leq 2\left|\rho^{k}\right|_{L^{\infty}}^{3 / 4}\left|\nabla u^{k-1}\right|_{L^{2}}\left|\nabla u_{t}^{k}\right|_{L^{2}}\left|\sqrt{\rho^{k}} u_{t}^{k}\right|_{L^{2}}^{1 / 2}\left|\nabla u_{t}^{k}\right|_{L^{2}}^{1 / 2} \\
& \leq C\left|\rho^{k}\right|_{L^{\infty}}^{3}\left|\nabla u^{k-1}\right|_{L^{2}}^{4}\left|\sqrt{\rho^{k}} u_{t}^{k}\right|_{L^{2}}^{2}+\varepsilon\left|\nabla u_{t}^{k}\right|_{L^{2}}^{2} \\
& \leq C \Phi_{K}^{9 / 2}+\varepsilon\left|\nabla u_{t}^{k}\right|_{L^{2}}^{2} ;
\end{aligned}
$$




$$
\begin{aligned}
& V_{2}=\int \rho^{k}\left|u^{k-1}\right|\left|\nabla u^{k-1}\right|\left|\nabla u^{k}\right|\left|u_{t}^{k}\right| \mathrm{d} x \\
& \leq\left|\rho^{k}\right|_{L^{\infty}}\left|u^{k-1}\right|_{L^{6}}\left|u_{t}^{k}\right|_{L^{6}}\left|\nabla u^{k-1}\right|_{L^{3}}\left|\nabla u^{k}\right|_{L^{3}} \\
& \leq\left|\rho^{k}\right|_{L^{\infty}}\left|\nabla u^{k-1}\right|_{L^{2}}\left|\nabla u_{t}^{k}\right|_{L^{2}}\left|\nabla u^{k-1}\right|_{L^{2}}^{1 / 2}\left|\nabla u^{k-1}\right|_{H^{1}}^{1 / 2}\left|\nabla u^{k}\right|_{L^{2}}^{1 / 2}\left|\nabla u^{k}\right|_{H^{1}}^{1 / 2} \\
& \leq C\left|\rho^{k}\right|_{L^{\infty}}^{2}\left|\nabla u^{k-1}\right|_{L^{2}}^{2}\left|\nabla u^{k-1}\right|_{L^{2}}\left|\nabla u^{k-1}\right|_{H^{1}}\left|\nabla u^{k}\right|_{L^{2}}\left|\nabla u^{k}\right|_{H^{1}}+\varepsilon\left|\nabla u_{t}^{k}\right|_{L^{2}}^{2} \\
& \leq \Phi_{K}^{2 \gamma+8}+\varepsilon\left|\nabla u_{t}^{k}\right|_{L^{2}}^{2} \\
& V_{3}=\int \rho^{k}\left|u^{k-1}\right|^{2}\left|\nabla^{2} u^{k}\right|\left|u_{t}^{k}\right| \mathrm{d} x \leq\left|\rho^{k}\right|_{L^{\infty}}\left|u^{k-1}\right|_{L^{6}}^{2}\left|\nabla^{2} u^{k}\right|_{L^{2}}\left|u_{t}^{k}\right|_{L^{6}} \\
& \leq\left|\rho^{k}\right|_{L^{\infty}}\left|\nabla u^{k-1}\right|_{L^{2}}^{2}\left|\nabla^{2} u^{k}\right|_{L^{2}}\left|\nabla u_{t}^{k}\right|_{L^{2}} \\
& \leq\left|\rho^{k}\right|_{L \infty}^{2}\left|\nabla u^{k-1}\right|_{L^{2}}^{4}\left|\nabla^{2} u^{k}\right|_{L^{2}}^{2}+\varepsilon\left|\nabla u_{t}^{k}\right|_{L^{2}}^{2} \\
& \leq \Phi_{K}^{2 \gamma+8}+\varepsilon\left|\nabla u_{t}^{k}\right|_{L^{2}}^{2} \\
& V_{4}=\int \rho^{k}\left|u^{k-1}\right|^{2}\left|\nabla u^{k}\right|\left|\nabla u_{t}^{k}\right| \mathrm{d} x \leq\left|\rho^{k}\right|_{L^{\infty}}\left|u^{k-1}\right|_{L^{6}}^{2}\left|\nabla u^{k}\right|_{L^{6}}\left|\nabla u_{t}^{k}\right|_{L^{2}} \\
& \leq\left|\rho^{k}\right|_{L^{\infty}}\left|\nabla u^{k-1}\right|_{L^{2}}^{2}\left|\nabla u^{k}\right|_{H^{1}}\left|\nabla u_{t}^{k}\right|_{L^{2}} \leq C\left|\rho^{k}\right|_{L^{\infty}}^{2}\left|\nabla u^{k-1}\right|_{L^{2}}^{4}\left|\nabla u^{k}\right|_{H^{1}}^{2}+\varepsilon\left|\nabla u_{t}^{k}\right|_{L^{2}}^{2} \\
& \leq \Phi_{K}^{2 \gamma+8}+\varepsilon\left|\nabla u_{t}^{k}\right|_{L^{2}}^{2} \\
& V_{5}=\int \rho^{k}\left|u_{t}^{k-1}\right|\left|\nabla u^{k}\right|\left|u_{t}^{k}\right| \mathrm{d} x \leq\left|\rho^{k}\right|_{L^{\infty}}^{1 / 2}\left|u_{t}^{k-1}\right|_{L^{6}}\left|\nabla u^{k}\right|_{L^{2}}\left|\sqrt{\rho^{k}} u_{t}^{k}\right|_{L^{3}} \\
& \leq\left|\rho^{k}\right|_{L^{\infty}}^{1 / 2}\left|\nabla u_{t}^{k-1}\right|_{L^{2}}\left|\nabla u^{k}\right|_{L^{2}}\left|\sqrt{\rho^{k}} u_{t}^{k}\right|_{L^{2}}^{1 / 2}\left|\sqrt{\rho^{k}} u_{t}^{k}\right|_{L^{6}}^{1 / 2} \\
& \leq\left|\rho^{k}\right|_{L^{\infty}}^{3 / 4}\left|\nabla u_{t}^{k-1}\right|_{L^{2}}\left|\nabla u^{k}\right|_{L^{2}}\left|\sqrt{\rho^{k}} u_{t}^{k}\right|_{L^{2}}^{1 / 2}\left|\nabla u_{t}^{k}\right|_{L^{2}}^{1 / 2} \\
& \leq\left|\rho^{k}\right|_{L^{\infty}}^{3}\left|\nabla u^{k}\right|_{L^{2}}^{4}\left|\sqrt{\rho^{k}} u_{t}^{k}\right|_{L^{2}}^{2}+\varepsilon\left|\nabla u_{t}^{k}\right|_{L^{2}}^{2}+\eta\left|\nabla u^{k-1}\right|_{L^{2}}^{2} \\
& \leq C \Phi_{K}^{9 / 2}+\varepsilon\left|\nabla u_{t}^{k}\right|_{L^{2}}^{2}+\eta\left|\nabla u^{k-1}\right|_{L^{2}}^{2} \\
& V_{6}=2 \zeta \int\left|w_{t}^{k}\right|\left|\nabla u_{t}^{k}\right| \mathrm{d} x \leq \zeta\left|w_{t}^{k}\right|_{L^{2}}^{2}+\zeta\left|\nabla u_{t}^{k}\right|_{L^{2}}^{2} \\
& V_{7}=\int\left|\nabla p^{k}\right|\left|u^{k-1}\right|\left|\operatorname{div} u_{t}^{k}\right| \mathrm{d} x \leq\left|\rho^{k}\right|_{L^{\infty}}^{\gamma-1}\left|\nabla \rho^{k}\right|_{L^{3}}\left|u^{k-1}\right|_{L^{6}}\left|\nabla u_{t}^{k}\right| L^{2} \\
& \leq C\left|\rho^{k}\right|_{L^{\infty}}^{2(\gamma-1)}\left|\nabla \rho^{k}\right|_{L^{3}}^{2}\left|\nabla u^{k-1}\right|_{L^{2}}^{2}+\varepsilon\left|\nabla u_{t}^{k}\right|_{L^{2}}^{2} \leq C \Phi_{K}^{2 \gamma}+\varepsilon\left|\nabla u_{t}^{k}\right|_{L^{2}}^{2} ; \\
& V_{8}=\int p^{\prime}\left(\rho^{k}\right) \rho^{k}\left|\operatorname{div} u^{k-1}\right|\left|\operatorname{div} u_{t}^{k}\right| \mathrm{d} x \leq C\left|\rho^{k}\right|_{L^{\infty}}\left|\nabla u^{k-1}\right|_{L^{2}}\left|\nabla u_{t}^{k}\right|_{L^{2}} \\
& \leq C\left|\rho^{k}\right|_{L^{\infty}}^{2 \gamma}\left|\nabla u^{k-1}\right|_{L^{2}}^{2}+\varepsilon\left|\nabla u_{t}^{k}\right|_{L^{2}}^{2} \leq C \Phi_{K}^{2 \gamma+1}+\varepsilon\left|\nabla u_{t}^{k}\right|_{L^{2}}^{2} .
\end{aligned}
$$

Substituting the above estimates into (63), and choosing $\varepsilon$ small enough, we have

$$
\frac{\mathrm{d}}{\mathrm{dt}} \int \rho^{k}\left|u_{t}^{k}\right|^{2} \mathrm{~d} x+\int\left|\nabla u_{t}^{k}\right| \mathrm{d} x \leq C \Phi_{K}^{2 \gamma+8}+\zeta\left|w_{t}^{k}\right|_{L^{2}}^{2}+\eta\left|\nabla u_{t}^{k-1}\right|_{L^{2}}^{2} .
$$


For a fixed $\tau \in(0, T)$, integrating the above inequality over $(\tau, t)$, we have:

$$
\int \rho^{k}\left|u_{t}^{k}\right|^{2} \mathrm{~d} x+\int_{\tau}^{t} \int\left|\nabla u_{t}^{k}\right| \mathrm{d} x \leq \int \rho^{k}\left|u_{t}^{k}\right|^{2}(\tau) \mathrm{d} x+C \int_{\tau}^{t} \Phi_{K}^{2 \gamma+8}+\int_{\tau}^{t}\left(\zeta\left|w_{t}^{k}\right|_{L^{2}}^{2}+\eta\left|\nabla u_{t}^{k-1}\right|_{L^{2}}^{2}\right) \mathrm{ds} .
$$

To estimate $\left|\sqrt{\rho^{k}} u_{t}^{k}(\tau)\right|_{L^{2}}^{2}$ as $\tau \rightarrow 0$, we multiply (47) by $u_{t}^{k}$, and integrate over $\Omega$. Then we have

$$
\int \rho^{k}\left|u_{t}^{k}\right|^{2} \mathrm{~d} x \leq 2 \int \rho^{k}\left|u^{k-1}\right|^{2}\left|\nabla u^{k}\right|^{2}+\rho^{-1}\left|-(\mu+\zeta) \Delta u^{k}-(\mu+\lambda-\zeta) \nabla \operatorname{div} u^{k}-2 \zeta \operatorname{rot} w^{k}+\nabla p^{k}\right|^{2} \mathrm{~d} x
$$

Hence, with the help of compatibility condition (6), we get:

$$
\limsup _{\tau \rightarrow 0}\left|\sqrt{\rho^{k}} u_{t}^{k}(\tau)\right|_{L^{2}}^{2} \leq C
$$

Considering this and letting $\tau \rightarrow 0$ in (64), we finally have:

$$
\int \rho^{k}\left|u_{t}^{k}\right|^{2} \mathrm{~d} x+\int_{0}^{t} \int\left|\nabla u_{t}^{k}\right| \mathrm{d} x \mathrm{~d} s \leq C \int_{0}^{t}\left(1+\Phi_{K}^{2 \gamma+8}\right) \mathrm{d} s+\int_{0}^{t}\left(\zeta\left|w_{t}^{k}\right|_{L^{2}}^{2}+\eta\left|\nabla u_{t}^{k-1}\right|_{L^{2}}^{2}\right) \mathrm{d} s
$$

Thanks to the estimate (65), (61), (57), (53), we get:

$$
\begin{aligned}
& \left|\sqrt{\rho^{k}} u_{t}^{k}\right|_{L^{2}}^{2}+\left|\sqrt{\rho^{k}} w_{t}^{k}\right|_{L^{2}}^{2}+\left|\nabla w^{k}\right|_{L^{2}}^{2}+\left|\nabla u^{k}\right|_{L^{2}}^{2}+\int_{0}^{t}\left(\left|\nabla u_{t}^{k}\right|_{L^{2}}^{2}+\left|\nabla w_{t}^{k}\right|_{L^{2}}^{2}\right) \mathrm{d} s \\
& \leq C \int_{0}^{t}\left(1+\Phi_{K}^{2 \gamma+8}\right) \mathrm{d} s+\left(\frac{\zeta}{2}+2 \eta\right) \int_{0}^{t}\left|\nabla u_{t}^{k-1}\right|_{L^{2}}^{2} \mathrm{~d} s .
\end{aligned}
$$

If $\eta$ is sufficient small such that $\frac{\zeta}{2}+2 \eta<\frac{3}{4} \zeta$, then from the recursive relation of $\left|\nabla u_{t}^{k}\right|_{L^{2}}^{2}$, it follows from that

$$
\int_{0}^{t}\left|\nabla u_{t}^{k}\right|_{L^{2}}^{2} \mathrm{~d} s \leq \zeta\left(1+\frac{3}{4}+\frac{9}{16}+\cdots\right) C \int_{0}^{t}\left(1+\Phi_{K}^{2 \gamma+8}\right) \mathrm{d} s .
$$

Thus we have

$$
\left|\sqrt{\rho^{k}} u_{t}^{k}\right|_{L^{2}}^{2}+\left|\sqrt{\rho^{k}} w_{t}^{k}\right|_{L^{2}}^{2}+\left|\nabla w^{k}\right|_{L^{2}}^{2}+\left|\nabla u^{k}\right|_{L^{2}}^{2}+\int_{0}^{t}\left(\left|\nabla u_{t}^{k}\right|_{L^{2}}^{2}+\left|\nabla w_{t}^{k}\right|_{L^{2}}^{2}\right) \mathrm{d} s \leq C \int_{0}^{t}\left(1+\Phi_{K}^{2 \gamma+8}\right) \mathrm{d} s .
$$

Finally, we recall from (18) that

$$
\left|\rho^{k}\right|_{H^{1} \cap W^{1, q_{0}}} \leq C \exp \left(C \int_{0}^{t}\left|\nabla u^{k-1}\right|_{H^{1} \cap \mathrm{w}^{1, q_{0}}} \mathrm{~d} s\right)
$$


for all $k, 1 \leq k \leq K$. To estimate $\left|\nabla u^{k}\right|_{W^{1, q_{0}}}$ for $1 \leq k \leq K$, we invoke the elliptic regularity result (36) and the estimate (51). If $3<q_{0}<6$, then we have

$$
\begin{aligned}
\left|\nabla u^{k}\right|_{W^{1, q_{0}}} \leq & C\left(\left|\rho^{k} u^{k}\right|_{L^{q_{0}}}+\left|\rho^{k} u^{k-1} \cdot \nabla u^{k}\right|_{L^{q_{0}}}+\left|\nabla w^{k}\right|_{L^{q_{0}}}+\left|\nabla p^{k}\right|_{L^{q_{0}}}+\left|\nabla u^{k}\right|_{L^{q_{0}}}\right) \\
\leq & C\left(\left|\rho^{k}\right|_{L^{\infty}}^{\left(2-\theta_{1}\right) / 2}\left|\sqrt{\rho^{k}} u_{t}^{k}\right|_{L^{2}}^{\theta_{1}}\left|\nabla u_{t}^{k}\right|_{L}^{1-\theta_{1}}+\left|\rho^{k}\right|_{L^{\infty}}\left|\nabla u^{k-1}\right|_{L^{6}}\left|\nabla u^{k}\right|_{L^{6 q_{0} /\left(6-q_{0}\right)}}\right. \\
& \left.+\left|\nabla p^{k}\right|_{L^{q_{0}}}+\left|\nabla u^{k}\right|_{H^{1}}\right) \\
\leq & C\left(\left|\rho^{k}\right|_{L^{\infty}}^{\left(2-\theta_{1}\right) /\left(1+\theta_{1}\right)}\left|\sqrt{\rho^{k}} u_{t}^{k}\right|_{L^{2}}^{2 \theta_{1} /\left(1+\theta_{1}\right)}+\left|\nabla u_{t}^{k}\right|_{L^{2}}^{2}+\Phi_{K}^{\gamma-1}\left|\nabla \rho^{k}\right|_{L^{q_{0}}}\right. \\
& \left.+\Phi_{K}^{2}\left|\nabla u^{k}\right|_{L^{2}}^{\theta_{2}}\left|\nabla u^{k}\right|_{W^{1, q_{0}}}^{1-\theta_{2}}+\Phi_{K}^{\gamma+5 / 2}\right) \\
\leq & C\left(\Phi_{K}^{\gamma+5 / 2}+\left|\nabla u_{t}^{k}\right|_{L^{2}}^{2}+\Phi_{K}^{2 / \theta_{2}+1}\right)+\frac{1}{2}\left|\nabla u^{k}\right|_{W^{1, q_{0}}}
\end{aligned}
$$

for some $\theta_{1}, \theta_{2} \in(0,1)$. Thus, we conclude that

$$
\left|\nabla u^{k}\right|_{W^{1, q_{0}}} \leq C\left(\Phi_{K}^{\max \left\{\gamma+5 / 2,2 / \theta_{2}+1\right\}}+\left|\nabla u_{t}^{k}\right|_{L^{2}}^{2}\right) .
$$

Similarly, from (28) and the estimate (50), we can deduce that,

$$
\begin{aligned}
& \left|\nabla w^{k}\right|_{W^{1, q_{0}}} \leq C\left(\left|\rho^{k} w_{t}^{k}\right|_{L^{q_{0}}}+\left|\rho^{k} u^{k-1} \cdot \nabla w^{k}\right|_{L^{q_{0}}}+\left|w^{k}\right|_{L^{q_{0}}}+\left|\nabla u^{k-1}\right|_{L^{q_{0}}}+\left|\nabla w^{k}\right|_{L^{q_{0}}}\right) \\
& \leq C\left(\left|\rho^{k}\right|_{L^{\infty}}^{\left(2-\theta_{3}\right)}\left|\sqrt{\rho^{k}} w_{t}^{k}\right|_{L^{2}}^{\theta_{3}}\left|\nabla w_{t}^{k}\right|_{L^{2}}^{1-\theta_{3}}+\left|\rho^{k}\right|_{L^{\infty}}\left|u^{k-1}\right|_{L^{6}}\left|\nabla w^{k}\right|_{L^{\left(\sigma_{0} /\left(6-q_{0}\right)\right.}}\left|\nabla w^{k}\right|_{L^{2}}+\left|\nabla w^{k}\right|_{H^{1}}\right) \\
& \leq C\left(\left|\rho^{k}\right|_{L^{\infty}}^{\left(2-\theta_{3}\right) /\left(1+\theta_{3}\right)}\left|\sqrt{\rho^{k}} w_{t}^{k}\right|_{L^{2}}^{2 \theta_{3} /\left(1+\theta_{3}\right)}+\left|\nabla w_{t}^{k}\right|_{L^{2}}^{2}+\Phi_{K}^{2}\left|\nabla w^{k}\right|_{L^{2}}^{\theta_{4}}\left|\nabla w^{k}\right|_{W^{1, q_{0}}}^{1-\theta_{4}}+\Phi_{K}^{5 / 2}\right) \\
& \leq C\left(\Phi_{K}^{5 / 2}+\left|\nabla w_{t}^{k}\right|_{L^{2}}^{2}+\Phi_{K}^{2 / \theta_{4}+1}\right)+\frac{1}{2}\left|\nabla w^{k}\right|_{W^{1, q_{0}}}
\end{aligned}
$$

for some $\theta_{3}, \theta_{4} \in(0,1)$. Thus, we deduce that

$$
\left|\nabla w^{k}\right|_{W^{1, q_{0}}} \leq C\left(\Phi_{K}^{\left.5 / 2,2 / \theta_{2}+1\right\}}+\left|\nabla w_{t}^{k}\right|_{L^{2}}^{2}\right) \text {. }
$$

Thanks to the estimates (68), (69), (67), and (66), we can easily show that there exists a small time $T_{1} \in(0, T)$ depending only on the parameters $C$ such that the following uniform estimate:

$$
\begin{aligned}
& \sup _{0 \leq t \leq T_{1}}\left(\left|\rho^{k}\right|_{H^{1} \cap W^{1, q_{0}}}+\left|\rho_{t}^{k}\right|_{L^{2} \cap L^{q_{0}}}+\left|\left(u^{k}, w^{k}\right)\right|_{D_{0}^{1} \cap D^{2}}+\left|\left(\sqrt{\rho^{k}} u_{t^{\prime}}^{k} \sqrt{\rho^{k}} w_{t}^{k}\right)\right|_{L^{2}}\right) \\
& +\int_{0}^{T_{1}}\left(\left|\left(u^{k}, w^{k}\right)\right|_{D^{2, q_{0}}}^{2}+\left|\left(u_{t}^{k}, w_{t}^{k}\right)\right|_{D_{0}^{1}}\right) d t \leq C .
\end{aligned}
$$

for all $k \geq 1$.

\subsection{Convergence}

We show that the approximate solutions converge to a solution to the original problem (1)-(4) in a strong sense. To prove this, we define:

$$
\bar{\rho}^{k+1}=\rho^{k+1}-\rho^{k}, \bar{u}^{k-1}=u^{k-1}-u^{k}, \quad \bar{w}^{k+1}=w^{k+1}-w^{k} .
$$


Then it follows from (45)-(47), we have

$$
\begin{aligned}
& \bar{\rho}_{t}^{i+1}+\operatorname{div}\left(\bar{\rho}^{i+1} u^{i}\right)+\operatorname{div}\left(\rho^{i} \bar{u}^{i}\right)=0, \\
& \rho^{k+1} \bar{w}_{t}^{k+1}+\rho^{k+1} u^{k} \cdot \nabla \bar{w}^{k+1}+4 \zeta \bar{w}^{k+1}-\mu^{\prime} \Delta \bar{w}^{k+1}-\left(\mu^{\prime}+\lambda^{\prime}\right) \nabla \operatorname{div} \bar{w}^{k+1} \\
& =\bar{\rho}^{k+1}\left(-w_{t}^{k}-u^{k} \cdot \nabla w^{k}\right)-\rho^{k} \bar{u}^{k} \cdot \nabla w^{k}+2 \zeta \operatorname{rot} \bar{u}^{k}, \\
& \rho^{k+1} \bar{u}_{t}^{k+1}+\rho^{k+1} u^{k} \cdot \nabla \bar{u}^{k+1}+\nabla \bar{p}^{k+1}-(\mu+\zeta) \Delta \bar{u}^{k+1}-(\mu+\lambda-\zeta) \nabla \operatorname{div} \bar{u}^{k+1} \\
& =\bar{\rho}^{k+1}\left(-u_{t}^{k}-u^{k} \cdot \nabla u^{k}\right)-\rho^{k} \bar{u}^{k} \cdot \nabla u^{k}+2 \zeta \operatorname{rot} \bar{w}^{k+1} .
\end{aligned}
$$

Multiplying (72) by $\bar{w}^{k+1}$, integrating over $\Omega$, using (45) and Young's inequality we have

$$
\begin{aligned}
& \frac{\mathrm{d}}{\mathrm{d} t} \int \rho^{k+1}\left|\bar{w}^{k+1}\right|^{2} \mathrm{~d} x+4 \zeta \int\left|\bar{w}^{k+1}\right|^{2} \mathrm{~d} x+\mu^{\prime} \int\left|\nabla \bar{w}^{-k+1}\right|^{2} \mathrm{~d} x \\
\leq & \int\left(\left|\bar{\rho}^{k+1}\right|\left|w_{t}^{k}\right|\left|\bar{w}^{k+1}\right|+\left|\bar{\rho}^{k+1}\right|\left|u^{k}\right|\left|\nabla w^{k}\right|\left|\bar{w}^{k+1}\right|+\rho^{k}\left|\bar{u}^{k}\right|\left|\nabla w^{k}\right|\left|\bar{w}^{k+1}\right|+2 \zeta\left|\nabla \bar{u}^{k}\right|\left|\bar{w}^{k+1}\right|\right) \mathrm{d} x .
\end{aligned}
$$

And thus we have

$$
\begin{aligned}
& \frac{\mathrm{d}}{\mathrm{d} t}\left|\sqrt{\rho^{k+1}} \bar{w}^{k+1}\right|_{L^{2}}^{2}+4 \zeta\left|\bar{w}^{k+1}\right|_{L^{2}}^{2}+\frac{\mu^{\prime}}{2}\left|\nabla \bar{w}^{k+1}\right|_{L^{2}}^{2} \\
\leq & C\left(\left|\nabla w_{t}^{k}\right|_{L^{2}}^{2}+\left|\nabla u^{k}\right|_{L^{2}}^{2}\left|\nabla w^{k}\right|_{H^{1}}^{2}\right)\left(\left|\bar{\rho}^{k+1}\right|_{L^{3 / 2}}^{2}+\left|\bar{\rho}^{k+1}\right|_{L^{2}}^{2}\right) \\
& +C\left|\rho^{k}\right|_{L^{\infty}}\left|\nabla w^{k}\right|_{L^{2}}\left|\nabla w^{k}\right|_{H^{1}}\left|\sqrt{\rho^{k}} \bar{u}^{k}\right|_{L^{2}}^{2}+\zeta\left|\nabla \bar{u}^{k}\right|_{L^{2}}^{2}+\zeta\left|\bar{w}^{k+1}\right|_{L^{2}}^{2}
\end{aligned}
$$

Multiplying (73) by $\bar{u}^{k+1}$, integrating over $\Omega$, using (45) and Young's inequality, we obtain

$$
\begin{aligned}
& \frac{\mathrm{d}}{\mathrm{d} t} \int \rho^{k+1}\left|\bar{u}^{k+1}\right|^{2} \mathrm{~d} x+(\mu+\zeta) \int\left|\nabla \bar{u}^{k+1}\right|^{2} \mathrm{~d} x \\
\leq & \left.\int\left|\bar{\rho}^{k+1}\right|\left|u_{t}^{k}\right|\left|\bar{u}^{k+1}\right|+\left|\bar{\rho}^{k+1}\right|\left|u^{k}\right|\left|\nabla u^{k}\right|\left|\bar{u}^{k+1}\right|+\rho^{k}\left|\bar{u}^{k}\right|\left|\nabla u^{k}\right|\left|\bar{u}^{k+1}\right|+\left|\bar{p}^{k+1}\right|\left|\nabla \bar{u}^{k+1}\right|+2 \zeta\left|\bar{w}^{k+1}\right|\left|\nabla \bar{u}^{k+1}\right|\right) \mathrm{d} x .
\end{aligned}
$$

And thus

$$
\begin{aligned}
& \frac{\mathrm{d}}{\mathrm{d} t}\left|\sqrt{\rho^{k+1}} \bar{u}^{k+1}\right|_{L^{2}}^{2}+\frac{\mu}{2}\left|\nabla \bar{u}^{k+1}\right|_{L^{2}}^{2} \\
\leq & \left.C\left|\nabla u_{t}^{k}\right|_{L^{2}}^{2}+\left|\nabla u^{k}\right|_{L^{2}}^{2}\left|\nabla u^{k}\right|_{H^{1}}^{2}+\left|\rho^{k}\right|_{L^{\infty}}^{\gamma-1}+\left|\rho^{k+1}\right|_{L^{\infty}}^{\gamma-1}\right)\left(\left|\bar{\rho}^{k+1}\right|_{L^{3 / 2}}^{2}+\left|\bar{\rho}^{k+1}\right|_{L^{2}}^{2}\right)+\zeta\left|\bar{w}^{k+1}\right|_{L^{2}}^{2} .
\end{aligned}
$$

On the other hand, observing that (71), we can easily prove that $\bar{\rho}^{k+1} \in L^{\infty}\left(0, T_{1} ; L^{3 / 2}\right)$. Hence, multiplying (71) by $\operatorname{sgn}\left(\bar{\rho}^{k+1}\right)\left|\bar{\rho}^{k+1}\right|^{1 / 2}$ and integrating over $\Omega$, we have

$$
\frac{\mathrm{d}}{\mathrm{d} t}\left|\bar{\rho}^{k+1}\right|_{L^{3 / 2}}^{3 / 2} \leq C\left|\nabla u^{k}\right|_{L^{\infty}}\left|\bar{\rho}^{k+1}\right|_{L^{3 / 2}}^{3 / 2}+C\left|\rho^{k}\right|_{H^{1}}\left|\nabla \bar{u}^{k}\right|_{L^{2}}\left|\bar{\rho}^{k+1}\right|_{L^{3 / 2}}^{1 / 2} .
$$

Multiplying the above inequality by $\left|\bar{\rho}^{k+1}\right|_{L^{3 / 2}}^{3 / 2}$ on both side, we have

$$
\left.\frac{\mathrm{d}}{\mathrm{d} t}\left|\bar{\rho}^{k+1}\right|_{L^{3 / 2}}^{2} \leq C\left|\nabla u^{k}\right|_{L^{\infty}}+\left|\rho^{k}\right|_{H^{1}}^{2}\right)\left|\bar{\rho}^{k+1}\right|_{L^{3 / 2}}^{2}+\varepsilon\left|\nabla \bar{u}^{k}\right|_{L^{2}}^{2} .
$$


Similarly, we get:

$$
\left.\frac{\mathrm{d}}{\mathrm{d} t}\left|\bar{\rho}^{k+1}\right|_{L^{2}}^{2} \leq C\left|\nabla u^{k}\right|_{L^{\infty}}+\left|\rho^{k}\right|_{L^{\infty}}^{2}+\left|\nabla \rho^{k}\right|_{L^{3}}^{2}\right)\left|\bar{\rho}^{k+1}\right|_{L^{2}}^{2}+\varepsilon\left|\nabla \bar{u}^{k}\right|_{L^{2}}^{2} .
$$

Combining (74)-(77), we obtain:

$$
\begin{array}{r}
\frac{\mathrm{d}}{\mathrm{d} t}\left(\left|\sqrt{\rho^{k+1}} \bar{w}^{k+1}\right|_{L^{2}}^{2}+\left|\sqrt{\rho^{k+1}} \bar{u}^{k+1}\right|_{L^{2}}^{2}+\left|\bar{\rho}^{k+1}\right|_{L^{3 / 2}}^{2}+\left|\bar{\rho}^{k+1}\right|_{L^{2}}^{2}\right) \\
+\left|\nabla \bar{w}^{k+1}\right|_{L^{2}}^{2}+\left|\nabla \bar{u}^{k+1}\right|_{L^{2}}^{2}+\left|\bar{w}^{k+1}\right|_{L^{2}}^{2} \\
\leq C\left|\sqrt{\rho^{k}} \bar{u}^{k}\right|_{L^{2}}^{2}+F_{\varepsilon}^{k}(t)\left(\left|\bar{\rho}^{k+1}\right|_{L^{3 / 2}}^{2}+\left|\bar{\rho}^{k+1}\right|_{L^{2}}^{2}\right)+\varepsilon\left|\nabla \bar{u}^{k}\right|_{L^{2}}^{2}
\end{array}
$$

for some function $F_{\varepsilon}^{k}(t)$ with $\int_{0}^{t} F_{\varepsilon}^{k}(\mathrm{~s}) \mathrm{ds} \leq C+C t$ for all $0 \leq t \leq T_{1}$ and $k \geq 1$.

Let us define

$$
\varphi^{k+1}(t)=C\left(\left|\sqrt{\rho^{k+1}} \bar{w}^{k+1}(t)\right|_{L^{2}}^{2}+\left|\sqrt{\rho^{k+1}} \bar{u}^{k+1}(t)\right|_{L^{2}}^{2}+\left|\bar{\rho}^{k+1}(t)\right|_{L^{3 / 2}}^{2}+\left|\bar{\rho}^{k+1}(t)\right|_{L^{2}}^{2}\right)
$$

and

$$
\psi^{k+1}(t)=\left|\nabla \bar{u}^{k+1}(t)\right|_{L^{2}}^{2}+\left|\nabla \bar{w}^{k+1}(t)\right|_{L^{2}}^{2}+\left|\bar{w}^{k+1}\right|_{L^{2}}^{2} .
$$

Then integrating $(78)$ over $(0, t) \subset\left(0, T_{1}\right)$, we have

$$
\varphi^{k+1}(t)+\int_{0}^{t} \psi^{k+1}(s) \mathrm{d} s \leq C \int_{0}^{t}\left(\varphi^{k}+\varepsilon \psi^{k}\right) \mathrm{d} s+\int_{0}^{t} F_{\varepsilon}^{k} \varphi^{k+1} \mathrm{~d} s
$$

which implies, by virtue of Gronwall's inequality, that

$$
\varphi^{k+1}(t)+\int_{0}^{t} \psi^{k+1}(s) \mathrm{d} s \leq C_{1} \exp \left(C_{2} t\right) \int_{0}^{t}\left(\varphi^{k}+\varepsilon \psi^{k}\right) \mathrm{d} s .
$$

Hence, choosing $\varepsilon>0$ and then $T_{*}>0$ so small that $4\left(T_{*}+\varepsilon\right) C_{1}<1, T_{*}<T_{1}$ and exp $\left(C_{2} T_{*}\right)<2$, we also deduce from Gronwall's inequality that for all $K \geq 1$,

$$
\sum_{k=1}^{K}\left(\sup _{0 \leq t \leq T_{*}} \varphi^{k+1}(t)+\int_{0}^{T_{*}} \psi^{k+1}(t) \mathrm{d} t\right)<\infty .
$$

Therefore, we conclude that $\left(\rho^{k}, u^{k}, w^{k}\right)$ converges to a limit $(\rho, u, w)$ in the following strong sense:

$$
\begin{aligned}
& \left(u^{k}, w^{k}\right) \rightarrow(u, w) \quad \text { in } L^{\infty}\left(0, T_{*} ; L^{2}\right) \cap L^{2}\left(0, T_{*} ; D_{0}^{1}\right) \\
& \text { and } \rho^{k} \rightarrow \rho \text { in } L^{\infty}\left(0, T_{*} ; L^{2}\right) .
\end{aligned}
$$

Now it is simple to check that $(\rho, u, w)$ is a weak solution to the original problem (1)-(4). Then, by virtue of the lower semi-continuity of norms, we deduce from the uniform bound $(70)$ that $(\rho, u, w)$ satisfies the following regularity estimate:

$$
\begin{aligned}
& \operatorname{esssup}\left(|\rho|_{H^{1} \cap w^{1, q_{0}}}+\left|\rho_{t}\right|_{L^{2} \cap L^{q_{0}}}+|(u, w)|_{D_{0}^{1} \cap D^{2}}+\mid\left(\sqrt{\rho} u_{t},\left.\sqrt{\rho} w_{t}\right|_{L^{2}}\right)\right. \\
& 0 \leq t \leq T_{*} \\
& \left.+\int_{0}^{T_{*}}(|u, w|)_{D^{2, q_{0}}}^{2}+\left(\left|u_{t}, w_{t}\right|\right)_{D_{0}^{1}}^{2}\right) \mathrm{d} t \leq C .
\end{aligned}
$$


The time-continuity of the solution $(\rho, u, w)$ can be proved by the same argument as in Section 2. This completes the proof of Proposition 3.1.

Here we should emphasize that the constant $C$ and the local existence time $T *$ in (79) do not depend on $\delta$ and the size of $\Omega$.

\section{Proof of theorem 1.1}

In this section, we complete the proof of Theorem 1.1.

Assume for the moment that $\Omega$ is a bounded domain with smooth boundary. Let $\left(\rho_{0}, u_{0}, w_{0}\right)$ be the given data satisfying (5). For each small $\delta>0$, let $\rho_{0}^{\delta}=\rho_{0}+\delta$ and let $\left(u_{0}^{\delta}, w_{0}^{\delta}\right) \in H_{0}^{1} \cap H^{2}$ be the unique smooth solution to the elliptic problem:

$$
\left\{\begin{array}{l}
-(\mu+\zeta) \Delta u_{0}^{\delta}-(\mu+\lambda-\zeta) \nabla \operatorname{div} u_{0}^{\delta}-2 \zeta \operatorname{rot} w_{0}^{\delta}+\nabla p\left(\rho_{0}^{\delta}\right)=\left(\rho_{0}^{\delta}\right)^{1 / 2} g_{1} \cdot \text { in } \Omega . \\
-\mu^{\prime} \Delta w_{0}^{\delta}-\left(\mu^{\prime}+\lambda^{\prime}\right) \nabla \operatorname{div} w_{0}^{\delta}-2 \zeta \operatorname{rot} u_{0}^{\delta}+4 \zeta w_{0}^{\delta}=\left(\rho_{0}^{\delta}\right)^{1 / 2} g_{2,}
\end{array}\right.
$$

Then by virtue of Proposition 3.1, there exist a time $T_{*} \in(0, T)$ and a unique strong solution $\left(\rho^{\delta}, u^{\delta}, w^{\delta}\right)$ in $\left[0, T_{*}\right] \times \Omega$ to the problem (1)-(4) with the initial data replaced by $\left(\rho_{0}^{\delta}, u_{0}^{\delta}, w_{0}^{\delta}\right)$. Notice that $\left(u_{0}^{\delta}, w_{0}^{\delta}\right) \rightarrow\left(u_{0}, w_{0}\right)$ in $H^{2}$ as $\delta \rightarrow 0,\left(\rho^{\delta}, u^{\delta}, w^{\delta}\right)$ satisfies the bound (44), and the constant $T_{*}, C$ are independent of $\delta$. Hence, following the same argument as in the proof of Theorem 2.1, we prove the existence and regularity of a strong solution to the original problem (1) - (4). Moreover, since the constant $C$ and the local existence $T_{*}$ in (44) are independent of the size of the domain, we also obtain the same existence and regularity results for unbounded domains by means of the domain expansion technique. Finally, the uniqueness can be proved by using the similar methods to the proof of the convergence in Section 3.

This completes the proof of Theorem 1.1.

\section{Acknowledgements}

The author was indebted to the referee for giving valuable suggestions which improve the presentation of the article.

\section{Competing interests}

The author declares that they have no competing interests.

Received: 4 December 2011 Accepted: 20 March 2012 Published: 20 March 2012

\section{References}

1. Eringen, AC: Theory of micropolar fluids. J Math Mech. 16, 1-18 (1966)

2. Lukaszewicz, G: Micropolar Fluid: Theory and Applications Modeling and Simulation in Science, Engineering and Technology. Birkhäuser, Boston (1999)

3. Yamaguchi, N: Existence of global strong solution to the micropolar fluid system in a bounded domain. Math Meth Appl Sci. 28, 1507-1526 (2005). doi:10.1002/mma.617

4. Lukaszewicz, G: On nonstationary flows of asymmetric fluids. Rendiconti Accademia Nazionale delle Scienze della dei XL Serie V. Memorie di Matematica. 12, 83-97 (1988)

5. Yuan, J: Existence theorem and blow-up criterion of strong solutions to the magneto-micropolar fluid equations. Math Meth Appl Sci. 31, 1113-1130 (2008). doi:10.1002/mma.967

6. Amirat, Y, Hamdache, K: Global weak solutions to a ferrofluid flow model. Math Meth Appl Sci. 31, 123-151 (2008). doi:10.1002/mma.896

7. Amirat, Y, Hamdache, K, Murat, F: Global weak solutions to equations of motion for magnetic fluids. J Math Fluid Mech. 10, 326-351 (2008). doi:10.1007/s00021-006-0234-6

8. Amirat, Y, Hamdache, K: Unique solvability of equations of motion for ferrofluids. Nonlinear Anal. 73, 471-494 (2010). doi:10.1016/j.na.2010.03.042

9. Mujaković, N: One-dimensional flow of a compressible viscous micropolar fluid: a global existence theorem. Glasnic matematićki. 33, 199-208 (1998)

10. Mujaković, N: Global in time estimates for one-dimensional compressible viscous micropolar fluid model. Glasnic matematički. 40, 103-120 (2005)

11. Mujaković, N: Non-homogeneous boundary value problem for one-dimensional compressible viscous micropolar fluid model: a global existence theorem. Math Inequal Appl. 12, 651-662 (2009)

12. Mujakovič, N: Nonhomogeneous boundary value problem for one-dimensional compressible viscous micropolar fluid model: regularity of the solution. Boundary Value Problems 2008, 15 (2008). Article ID 189748 
13. Dražič, l, Mujakovič, N: Approximate solution for 1-D compressible viscous micropolar fluid model in dependence of initial conditions. Int J Pure Appl Math. 42, 535-540 (2008)

14. Cowin, SC: The theory of polar fluids. Adv Appl Mech. 14, 279-347 (1974)

15. Easwaran, CV, Majumdar, SR: A uniqueness theorem for compressible micropolar flows. Acta Mech. 68, 185-191 (1987). doi:10.1007/BF01190882

16. Ramkissoon, H: Boundary value problems in microcontinuum fluid mechanics. Quart Appl Math. 42, 129-141 (1984)

17. Galdi, GP, Rionero, S: A note on the existence and uniqueness of solutions of the micropolar fluid equations. Internat J Engrg Sci. 15, 105-108 (1977). doi:10.1016/0020-7225(77)90025-8

18. Chen, MT: Global strong solutions for the viscous, micropolar, compressible flow. J Part Diff Equ. 24, 158-164 (2011)

19. Chen, MT: Global existence of strong solutions to the micro-polar, compressible flow with density-dependent viscosities. Boundary Value Problems. 2011, 13 (2011). doi:10.1186/1687-2770-2011-13

20. Amirat, $Y$, Hamdache, $K$ : Weak solutions to the equations of motion for compressible magnetic fluids. J Math Pure Appl. 91, 433-467 (2009)

21. Matsumura, A, Nishida, T: The initial value problem for the equations of motion of viscous and heatconductive gases. J Math Kyoto Univ. 20, 67-104 (1980)

22. Lions, PL: Mathematical Topics in Fluid Mechanics. In Compressible models, vol. 2,Oxford University Press, New York (1998)

23. Feireisl, E, Novotný, A, Petzeltová, H: On the existence of globally defined weak solutions to Navier-Stokes equations of isentropic compressible fluids. J Math Fluid Dyn. 3, 358-392 (2001)

24. Choe, HJ, Kim, H: Strong solutions of the Navier-Stokes equations for isentropic compressible fluids. J Diff Equ. 190 504-523 (2003). doi:10.1016/50022-0396(03)00015-9

25. Cho, Y, Kim, H: Existence results for viscous polytropic fluids with vacuum. J Diff Equ. 228, 377-411 (2006). doi:10.1016/j. jde. 2006.05 .001

26. Cho, Y, Choe, HJ, Kim, H: Unique solvability of the initial boundary value problems for compressible viscous fluids. J Math Pures Appl. 83, 243-275 (2004)

27. Hoff, D, Serre, D: The failure of continous dependence on initial data for the Navier-Stokes equations of compressible flow. SIAM J Appl Math. 51, 887-898 (1991). doi:10.1137/0151043

28. Valli, A: An existence theorem for compressible viscousfluids. Ann Mat Pura Appl. 130, 197-213 (1982). doi:10.1007/ BF01761495

29. Valli, A: Periodic and stationary solutions for compressible Navier-Stokes equations via a stability method. Ann Scuola Norm Sup Pisa Cl Sci. 10, 607-647 (1983)

30. DiPerna, RJ, Lions, PL: Ordinary differential equations, transport theory and Sobolev space. Invent Math. 98, 511-547 (1989). doi:10.1007/BF01393835

31. Temam, R: Navier-Stokes Equation: Theory and Numerical Analysis. North-Holland, Amsterdam (1984)

32. Galdi, GP: An introduction to the mathematical theory of the Navier-Stokes equations. Springer-Verlag, New York (1994)

doi:10.1186/1687-2770-2012-32

Cite this article as: Chen: Unique solvability of compressible micropolar viscous fluids. Boundary Value Problems 2012 2012:32

\section{Submit your manuscript to a SpringerOpen ${ }^{\circ}$} journal and benefit from:

- Convenient online submission

- Rigorous peer review

- Immediate publication on acceptance

- Open access: articles freely available online

- High visibility within the field

- Retaining the copyright to your article

Submit your next manuscript at $\boldsymbol{s p r i n g e r o p e n . c o m ~}$ 